Artvin Çoruh Üniversitesi

Orman Fakültesi Dergisi

ISSN:2146-1880, e-ISSN: 2146-698X

YII: 2018, Cilt: 19, Sayı:1, Sayfa: 93-113

\title{
Karadağ florası (Yusufeli, Artvin-Türkiye)
}

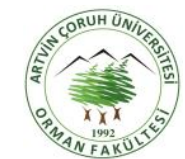

ofd.artvin.edu.t
Artvin Coruh University

Journal of Forestry Faculty

ISSN:2146-1880, e-ISSN: 2146-698X

Year: 2018, Vol: 19, Issue: 1, Pages: 93-113

\section{The flora of Karadağ (Yusufeli, Artvin-Turkey)}

\author{
Özgür EMINAĞAOĞLU1 ${ }^{1}$, Hayal AKYILDIRIM BEĞEN², Güven AKSU ${ }^{1}$ \\ ${ }^{1}$ Artvin Çoruh Üniversitesi, Orman Fakültesi, Orman Mühendisliği Bölümü, Artvin, Türkiye \\ ${ }^{2}$ Artvin Çoruh Üniversitesi, Sağlık Hizmetleri Meslek Yüksekokulu, Artvin, Türkiye
}

\section{Eser Bilgisi / Article Info}

Araştırma makalesi / Research article

DOI: 10.17474/artvinofd.352425

Sorumlu yazar / Corresponding author Özgür EMINAĞAOĞLU

e-mail: oeminagaoglu@artvin.edu.tr ORCID: 0000-0003-0064-0318

Geliş tarihi / Received

14.11.2017

Düzeltme tarihi / Received in revised form

21.03.2018

Elektronik erişim / Online available

30.04.2018

Anahtar kelimeler:

Artvin

Endemik

Flora

Karadağ

Kolşik

Yusufeli

\section{Keywords:}

Artvin

Endemic

Flora

Karadag

Colchic

Yusufeli

\section{Özet}

Bu çalışma, Artvin ili Yusufeli ilçesinde bulunan Karadağ Florası'nın tespit edilmesi amacıyla 2014 ve 2015 yılları arasında yapılmıştır. Araştırma alanı, Davis'in grid sistemine göre A9 karesinde yer almakta olup Holarktik bölgenin, Avrupa-Sibirya flora alanının Öksin kesiminin Kolşik altkesiminde ve 612-2388 m yükseltiler arasında yer almaktadır. Bu araştırmada 91 familya, 331 cinse ilişkin toplam 493 adet takson saptanmıştır. Bunlardan 1 takson Lycopodiophyta, 18 takson Pteridophyta, 474 takson ise Magnoliophyta bölümüne ilişkindir. Magnoliophyta bölümüne ilişkin taksonlardan 7'si Pinophytina, 467'si Magnoliophytina alt bölümünde bulunmaktadır. Araştırma alanında en fazla takson içeren 5 familya sırasıyla; Asteraceae (60 takson), Poaceae (55 takson), Fabaceae (32 takson), Lamiaceae (28 takson) ve Rosaceae (21 takson). Bunların fitocoğrafik bölgelere göre dağılımları ise şöyledir: 130'u (\%26.4) Avrupa-Sibirya, 93'ü (\%18.9) İran-Turan, 24'ü (\%4.9) Akdeniz ve coğrafi bölgesi bilinmeyip birden fazla bölgede yayılış gösterenler 246 adettir $(\% 49,8)$. 352 taksonun hayat formlarına göre dağılım ve oranları sırasıyla şöyledir: Hemikriptofitler 121 takson (\%24.5), kriptofitler 87 takson (\%17.6), terofitler 51 takson (\%10.3), fanerofitler 49 takson (\%9.9) ve kamefitler 44 takson (\%8.9) ve hayat formları belirlenemeyen 141 adet takson bulunmaktadır. Araştırma alanında 40 adet endemik ve 50 adet de endemik olamayan nadir bitki türü saptanmış olup endemizim oranı \%8.1'dir. IUCN tehlike kategorilerine göre endemik ve endemik olmayan 90 adet nadir taksonun tehlike durumu değerlendirilmiştir.

\begin{abstract}
This study was carried out between the years of 2014 and 2015 in order to determine the flora of Karadag in the province of Yusufeli, Artvin. The research area under review takes place in Colchic province of Euro-Siberian floristic area of Holarctic region and is found in the A9 square according to grid system of Davis and lies between 612 and $2388 \mathrm{~m}$ elevation above the sea level. In this study, total 493 plant taxa belonging to 91 families and 331 genera were determined. 1 of them belong to Lycopodiophyta, 18 of them is from Pteridophyta, the remaining 474 taxa were Magnoliophyta which included 7 taxa from Pinophytina and 467 taxa from Magnoliophytina. The largest families recorded were as follows: Asteraceae (60 taxon), Poaceae (55 taxon), Fabaceae (32 taxon), Lamiaceae (28 taxon), Rosaceae (21 taxon), Boraginaceae (19 taxon), Brassicaceae (18 taxon), Apiaceae (18 taxon), Plantaginaceae (12 taxon), Caryophyllaceae (11 taxon), Caprifoliaceae (9 taxon) and Orobanchaceae (9 taxon). The distribution of the taxa according to the phytogeographical regions was as follows: Avrupa-Sibirya with 130 taxa $(26,4 \%)$, Iran-Turan with 93 taxa (18.9\%), the Mediterranean with 24 taxa $(4,9 \%)$ and multiregional or of unknown phytogeographic origin with 246 taxa $(49.8 \%)$. The life form spectrum of 352 taxa was as follows: Hemicryptophytes 121 taxa (24.5\%), cryptophytes 87 taxa (17.6\%), therophytes 51 taxa $(10.3 \%)$, phanerophytes 49 taxa $(9,9 \%)$ and chamaephytes 44 taxa $(8.9 \%)$. Endemism ratio is $8.1 \%$ and included 40 endemic taxa and 50 non-endemic rare plants. The endemic plants and non-endemic rare plants (90 taxa) in the study area have been classified according to IUCN threat catagories.
\end{abstract}

\section{Giriş}

Çalışma alanı olan Karadağ, Türkiye'nin kuzeydoğusunda, Artvin ili sınırları içinde yer almaktadır. Artvin; dünyanın biyolojik çeşitlilik açısından en zengin ve aynı zamanda tehlike altındaki en önemli "34 Biyoçeşitlilik Sıcak Noktası"ndan biridir; aynı zamanda, Batı Avrasya'daki Üçüncü Zaman’a ait ormanların en önemli sığınak ve relikt alanıdır. Bölgenin koruma açısından önemini kabul eden Dünya Doğayı Koruma Vakfı (WWF) da, dünya üzerinde korumada öncelikli "200 Ekolojik Bölge"den biri olarak ilan etmiştir. Ayrıca Türkiye'nin tek biyosfer rezerv alanı yine Artvin ili sınırları içerisinde yer almaktadır. Artvin; dünya üzerinde endemik ve nadir bitkilerin çok sayıda bulunduğu koruma değeri yüksek " 45 Bitkisel Çeşitlilik 
Merkezinden biri olan "Kuzeydoğu Anadolu Bitkisel Çeşitlilik Merkezi" içerisinde yer almaktadır. Türkiye'de 154 familya ait, 1220 cins, 9753 tür, 1985 alttür, 858 varyete, 253 melez tür ve 5 melez alttür olamak üzere toplam 11466 takson doğal olarak yayılış göstermektedir. Bu sayıya yabancı türler ve tarımda kullanılanlar da eklendiğinde toplam takson sayısı 11707'ye ulaşmaktadır. Türkiye Florası'nın diğer bir önemli özelliği de çok sayıda endemik takson içermesidir. Ülkemizdeki endemik bitki takson sayısı 3649, endemizm oranı ise \%31,82' dir (Güner ve ark. 2012; Eminağaoğlu ve ark. 2015a). Artvin ili florası 137 familya, 761 cinse ilişkin olamak üzere 2727 taksona sahiptir. Bu bitki taksonlarının 198'iendemik olmak üzere toplam 500'ü nadir bitki olup endemizm oranı \%7.2'dir (Eminağaoğlu ve ark. 2015a).

Araştırma alanına yakın bölgelerde; Anşin (1979, 1982), Düzenli (1988), Vural (1996), Eminağaoğlu (1996, 2002), Eminağaoğlu ve Anşin (2002, 2003, 2004), Eminağaoğlu ve ark. (2008), Eminağaoğlu ve Akpulat (2010), Eminağaoğlu ve ark. (2012), Eminağaoğlu (2015) tarafından floristik çalışmalar gerçekleştirilmiştir. Türkiye Florası (Davis, 1965-1985)'na göre birçok türk ve yabancı araştırmacı bölgeyi ziyaret etmiş ve bitki örneği toplamışlardır. Bunlar arasında Albov, Andronakai, Anflin, Bornmüller, Davis, Düzenli, Grossheim, Güner, HandelMazetti, Henderson, Koch, Komarov, Krause, Louis, Maleev, Mirov, Radde, Sauer, Stainton, Vural, Vvedensky ve Woronow sayılabilir. Bunlardan özellikle Anşin Davis, Grossheim, Sauer, Vvedensky ve Woronow Yusufeli ilçesinden bitki toplamışlardır (Davis 1965-1985; Mirov 1967; Davis ve ark. 1988; Güner ve ark. 2000).

Araştırma alanını kapsayan bir floristik çalışmanın bulunmaması, coğrafik konumu, topoğrafik yapısı, kendine özgü iklimi ve Avrupa-Sibirya ve Iran-Turan flora alanları arasında geçiş bölgesinde olması da dikkate alınarak, bu çalışma ile Karadağ'ın sahip olduğu floristik yapının belirlenmesi ve floristik açıdan yeni olabilecek taksonların saptanması, Karadağ ve çevresinde bulunan endemik ve nadir bitkilerin tespiti ve bu çalışmadan elde edilecek verilerle Artvin Çoruh Üniversitesi Herbaryumu'na (ARTH) katkı sağlanması amaçlanmıştır.

\section{MATERYAL ve YÖNTEM}

\section{Çalışma Alanının Coğrafik Özellikleri}

Araştırma alanı; Davis'in grid sistemine göre A9 karesinde yer almakta olup Artvin ilinin Yusufeli ilçesi sınırları içerisinde bulunup, alan il merkezine $77 \mathrm{~km}$ ilçe merkezine ise $5 \mathrm{~km}$ uzaklıktadır. Alan, Holarktik bölgenin, AvrupaSibirya flora alanının Öksin kesiminin Kolşik alt kesiminde yer almakta ve 600-2388 m yükseltiler arasında bulunmaktadır; engebeli bir topoğrafya ya sahip olduğundan örtüsüz çorak alanlar geniş yer kaplamaktadır (Eminağaoğlu ve ark. 2015b) (Şekil 1).

\section{Çalışma Alanının İklimi}

Artvin ilinin Yusufeli ilçesi iklim özelliklerinin belirlenmesinde ise Artvin Meteoroloji i̇stasyonunun uzun süreli (2003-2013 yılları arası) gözlem değerleri kullanılmıştır (DMi, 2015). Yıllık ortalama sıcaklık $12.6{ }^{\circ} \mathrm{C}$, yıllık ortalama yüksek sıcaklık $40.7{ }^{\circ} \mathrm{C}$, yıllık ortalama düşük sıcaklık - $9.1{ }^{\circ} \mathrm{C}^{\prime}$ dir. Yılın en sıcak ayı $40.7^{\circ} \mathrm{C}$ ile temmuz ayı, yılın en soğuk ayı ise $-9,9{ }^{\circ} \mathrm{C}$ ile aralık ayıdır. Yıllık ortalama yağış $741.5 \mathrm{~mm}$ olup, yııın en yağışlı ayı $68,8 \mathrm{~mm}$ ile şubat ayıdır. Mevsimler itibariyle yağış rejimi ilkbahardan yaza doğru hızla azalmaktadır (Çizelge 1). En yağışlı mevsim kış, en kurak mevsim yazdır (Şekil 2). 


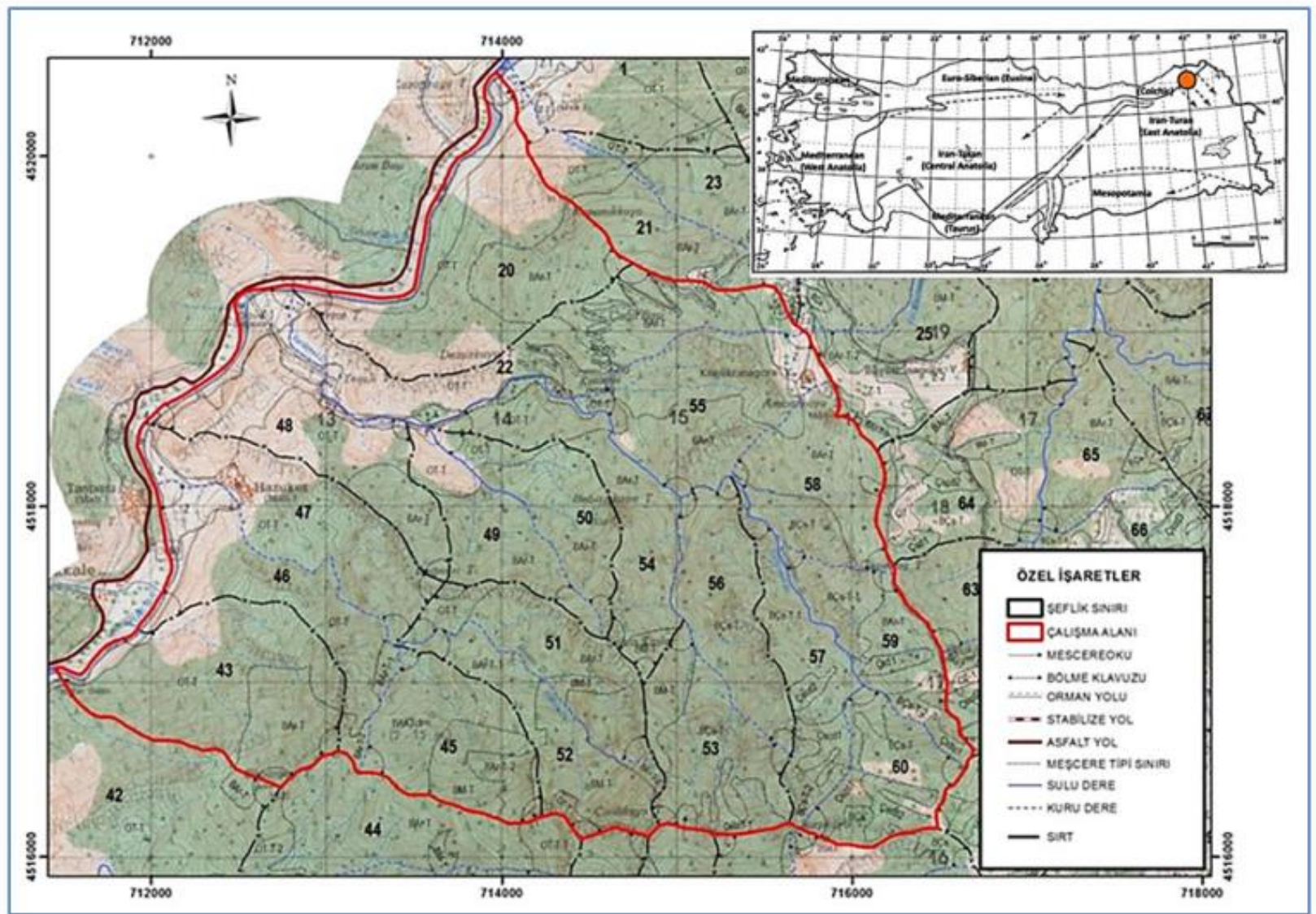

Şekil 1. Araştırma alanının coğrafi konumu, fitocoğrafik bölgelere göre durumu, topografik ve meşcere haritası (Eminağaoğlu ve ark. 2016)

Çizelge 1. Yusufeli ilçesi sıcaklık verileri

\begin{tabular}{|c|c|c|c|c|c|c|c|c|c|c|c|c|c|}
\hline & & & & & A & $Y$ & L & $A$ & $\mathrm{R}$ & & & & \\
\hline Meteorolojik Elemanlar & 1 & 2 & 3 & 4 & 5 & 6 & 7 & 8 & 9 & 10 & 11 & 12 & Yıllık \\
\hline Ortalama Sıcaklık (oC) & 1.3 & 2.6 & 8 & 14.9 & 19.1 & 22.8 & 25.7 & 26 & 22.2 & 15.8 & 8.4 & 2.8 & 14.13 \\
\hline Max. Sıcaklık (OC) & 15.8 & 20.0 & 25.5 & 32.0 & 36.9 & 38.5 & 41.8 & 43.8 & 38.8 & 34.4 & 23.1 & 16.3 & 43.8 \\
\hline Min. Sıcaklık (oC) & -16.5 & -12.5 & -13.3 & 0.3 & 2.7 & 9.0 & 10.8 & 12.3 & 7.6 & 0.0 & -5.8 & -11.0 & -16.5 \\
\hline Toplam Yağış Ort. (mm) & 21.6 & 20.2 & 19.6 & 28.2 & 29.6 & 33.6 & 21.7 & 12.6 & 12.0 & 23.1 & 25.8 & 27.4 & 275.4 \\
\hline Max. Yağış (mm) & 33.2 & 19.0 & 18.0 & 24.5 & 28.8 & 21.2 & 56.0 & 18.8 & 18.3 & 43.9 & 32.1 & 47.2 & 56 \\
\hline
\end{tabular}

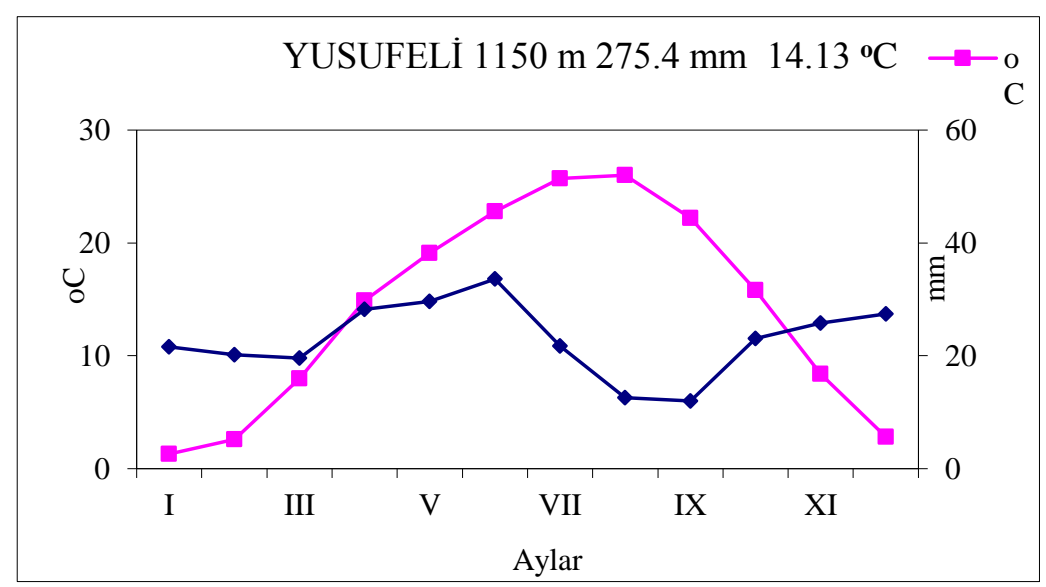

Şekil 2. Walter yöntemine göre Yusufeli su bilançosu

। 


\section{Materyal}

Floristik içeriğin saptanması amacıyla 2014-2015 yılları arası vejetasyon periyodu içerisinde yapılan floristik geziler sırasında toplanan bitki örneklerinde, teşhis ve tanımada gerekli olan meyve, çiçek, yaprak (otsularda dip yaprak), toprak altı kısmı (rizom, yumru, soğan v.b.), tomurcuk gibi vejetatif ve generatif organların bulunmasına dikkat edilmiştir.

Toplanan bitki örnekleri için mevki, yetişme yeri özellikleri, yükselti, toplama tarihi gibi bilgiler kaydedilmiştir. Toplanan bu örnekler, herbaryum tekniğine uygun olarak kurutulmuş, binoküler stereo zoom mikroskop altında teşhis edilmiş, kartonlara yapıştırılmış, etiketlenmiş ve adlandırılmıştır

Çalışma alanında 13 farklı istasyondan bitki toplanmış olup bu noktaların listesi aşağıdaki gibidir:

L1. A9-Artvin, Yusufeli, Karadağ,Çeltikdüzü, 4047'29.96 $\mathrm{N}, 41^{\circ} 31^{\prime} 10.03 \mathrm{E}, 636 \mathrm{~m}$.

L2. A9-Artvin, Yusufeli, Karadağ,Çeltikdüzü, $40^{\circ} 47^{\prime} 21.27$ $\mathrm{N}, 41^{\circ} 31^{\prime} 27.20 \mathrm{E} 688 \mathrm{~m}$.

L3. A9-Artvin, Yusufeli, Karadağ,Çeltikdüzü, $40^{\circ} 47^{\prime} 42.96$ $\mathrm{N}, 41^{\circ} 32^{\prime} 47.30$ E $1252 \mathrm{~m}$.

L4. A9-Artvin, Yusufeli, Karadağ,Çeltikdüzü, $40^{\circ} 47^{\prime} 25.47$ $\mathrm{N}, 41^{\circ} 34^{\prime} 04.77 \mathrm{E} 1480 \mathrm{~m}$.

L5. A9-Artvin, Yusufeli, Karadağ,Çeltikdüzü, $40^{\circ} 46^{\prime} 36.76$ $\mathrm{N}, 41^{\circ} 34^{\prime} 57.92$ E $1572 \mathrm{~m}$.

L6. A9-Artvin, Yusufeli, Karadağ,Çeltikdüzü, $40^{\circ} 46^{\prime} 01.99$ $\mathrm{N}, 41^{\circ} 35^{\prime} 28.63 \mathrm{E} 1737 \mathrm{~m}$.

L7. A9-Artvin, Yusufeli, Karadağ,Çeltikdüzü, $40^{\circ} 45^{\prime} 39.40$ $\mathrm{N}, 41^{\circ} 35^{\prime} 50.07$ E $2094 \mathrm{~m}$.

L8. A9-Artvin, Yusufeli, Karadağ,Çeltikdüzü, $40^{\circ} 45^{\prime} 37.40$ $\mathrm{N}, 41^{\circ} 36^{\prime} 16.10$ E $2254 \mathrm{~m}$.

L9. A9-Artvin, Yusufeli, Karadağ, Çeltikdüzü, $40^{\circ} 46^{\prime} 05.98$ $\mathrm{N}, 41^{\circ} 36^{\prime} 46.18$ E $2380 \mathrm{~m}$.

L10. A9-Artvin, Yusufeli, Karadağ,Çeltikdüzü, $40^{\circ} 46^{\prime} 39.62$ $\mathrm{N}, 41^{\circ} 38^{\prime} 35.51 \mathrm{E} 1813 \mathrm{~m}$.

L11. A9-Artvin, Yusufeli, Karadağ,Çeltikdüzü, $40^{\circ} 47^{\prime} 33.51$ $\mathrm{N}, 41^{\circ} 37^{\prime} 46.86 \mathrm{E} 1471 \mathrm{~m}$.

L12. A9-Artvin, Yusufeli, Karadağ,Çeltikdüzü, $40^{\circ} 47^{\prime} 16.11$ $\mathrm{N}, 41^{\circ} 36^{\prime} 39.23$ E $1914 \mathrm{~m}$.

L13. A9-Artvin, Yusufeli, Karadağ,Çeltikdüzü, $40^{\circ} 46^{\prime} 36.96$

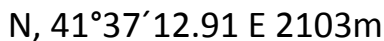

\section{Yöntem}

Kurutularak herbaryum materyali haline getirilen bitki örneklerinin adlandırımasında temel kaynak "Flora of Turkey and the East Eagean Islands" (Davis 1965-85; Davis ve ark. 1988; Güner ve ark. 2000) adlı eser olmakla birlikte Flora USSR (Komarov, 1934-78), Flora Kavkaza (Grossheim, 1939-1967), Akkemik (2014), Eminağaoğlu $(2012,2015)$ gibi flora kaynaklarından, renkli resimli çiçek atlaslarından da yararlanılmıştır.

Teşhis için hazırlanan kurutulmuş bitki örnekleri Nikon SMZ 1000 marka binoküler stereo zoom mikroskop kullanılarak teşhis anahtarlarında sahip olduğu morfolojik karakterlere göre (tohum, meyve, çiçek, yaprak, tüy..) tanımlamaları yapılıp, isimleri Türkiye Bitkileri Listesi (Güner ve ark. 2012) kontrol edilerek teşhisleri tamamlanmıştır.

Saptanan taksonlara ilişkin kategoriler, sistematik liste halinde verilirken Lycopodiophyta, Pteridophyta bölümüne ve Magnoliophyta bölümünün Pinophytina ve Magnoliophytina alt bölümüne ait taksonların sistematiği Davis (1965-1988) ve Güner ve ark. (2000)'e göre verilmiştir. Sistematik dizinde tür, alttür ve varyete, yetişme yeri özellikleri, yükselti, toplama tarihi, fitocoğrafik bölge, egzotik, kozmopolit ve endemizm durumu verilmiştir.

Taksonların Risk Kategorilerine göre değerlendirilmesinde; IUCN (2017), Red List of The Endemic Plants of The Caucasus (Ekim ve ark. 2014) ve Türkiye Bitkileri Kırmızı Kitabı (Ekim ve ark. 2000)'ndan yararlanılmıştır. Taksonların Hayat Formları Raunkiær (1934)'e göre değerlendirilmiştir. Ayrıca sistematik dizinde yazılan bitki isimleri The Plant List kullanılarak güncellenmiştir (URL-II).

\section{BULGULAR}

Arazi çalışmaları sırasında 1900 bitki örneğinin teşhisi sonucunda 91 familya, 331 cinse ilişkin toplam 493 adet takson saptanmıştır. Bunlardan 1 takson Lycopodiophyta, 18 takson Pteridophyta, 474 takson ise Magnoliophyta bölümüne aittir. Teşhis edilen taksonların sistematik dizini aşağıda verilmiştir: 
Sistematik Dizin

\section{LYCOPODIOPHYTA}

1. LYCOPODIACEAE

Lycopodium alpinum $\mathrm{L}$.

Alpin yamaçlar, 08.08.2014, L7, O.Emin 22373\&Aksu, Crp.

PTERIDOPHYTA

2. EQUISETACEAE

Equisetum hyemale $\mathrm{L}$.

Yol kenarı, 02.04.2015, L1, ;O.Emin 22575\&Aksu, LC, Crp.

E. ramosissimum Desf.

Su kenarı, 17.05.2015, L3, O.Emin 22610\&Aksu, Crp.

E. fluviatile L.

Sulak alan, 26.04.2015, L3, O.Emin 22375\&Aksu, LC, Crp.

E. arvense $\mathrm{L}$.

Dere kenarı, 02.04.2015, L5, O.Emin 22576\&Aksu, Crp.

E. telmateia Ehrh.

Dere kenarı, 02.04.2015, L3, O.Emin 22577\&Aksu, LC, Crp.

\section{OSMUNDACEAE}

Osmunda regalis $\mathrm{L}$.

Dere kenarı, 08.08.2014, L3, O.Emin 22374\&Aksu, Crp.

\section{PTERIDACEAE}

Adiantum capillus-veneris L.; Taş yamaçlar, 08.08.2014, L5, O.Emin 22375\&Aksu, LC, Euro-Sib., Crp.

\section{DENNSTAEDTIACEAE}

Pteridium aquilinum (L.) Kuhn.

Orman içi açık alan, 17.05.2015, L6, O.Emin 22611\&Aksu,

Crp.

\section{THELYPTERIDACEAE}

Thelypteris limbosperma (All.) H.P.Fuchs; Ormanı içi, dere kenarı, 08.08.2014, L5, O.Emin 22376\&Aksu, Crp.

\section{ASPLENIACEAE}

Asplenium trichomanes $\mathrm{L}$.

Orman içi açık alan, 02.04.2015, L1, O.Emin 22578\&Aksu, Crp.

A. septentrionale (L.) Hoffm.; Kaya kenarı, nemli alan, 02.04.2015, L1, O.Emin 22579\&Aksu, Crp.

A. scolopendrium L.; Taşlı ve nemli toprak, gölgeli alan, açık alan, 08.08.2014, L5, O.Emin 22377\&Aksu, Crp.

\section{ATHYRIACEAE}

Athyrium filix-femina (L.) Roth.; Orman içi nemli alan, 08.08.2014, L5, O.Emin 22378\&Aksu, Crp.

\section{ASPIDIACEAE}

Polystichum lonchitis (L.) Roth; Çalıık, taşlık-kayalık alan, 17.05.2015, L7, O.Emin 22612\&Aksu, Crp.

Dryopteris abbreviata (DC.) Newman; Orman içi açık alan, 08.08.2014, L4, O.Emin 22379\&Aksu, VU, Eux., Crp. D. dilatata (Hoffm.) A. Gray

Orman içi açık alan, 08.08.2014, L7, O.Emin 22380\&Aksu, Crp.
D. aemula (Aiton) Kuntze; Orman altı, nemli alan, 08.08.2014, L4, O.Emin 22381\&Aksu, Crp.

\section{POLYPODIACEAE}

Polypodium vulgare L. subsp. vulgare; Yol kenarı, kayalık alanlar, 08.08.2014, L3, O.Emin 22382\&Aksu, Crp.

\section{MAGNOLIOPHYTA}

\section{PINOPHYTINA}

\section{PINACEAE}

Abies nordmanniana (Stev.) Spach subsp. nordmanniana Picea orientalis ile birlikte, 17.05.2015, L7, O.Emin 22613\&Aksu, LC, Eux., Php.

Picea orientalis (L.) Peterm.

Yol kenarı, 17.05.2015, L6, O.Emin 22614\&Aksu, Eux., Php.

Pinus sylvestris L. var. hamata Steven.

Yol kenarı, 28.07.2015, L5, O.Emin 22784\&Aksu, Euro-Sib, Php.

\section{CUPRESSACEAE}

Juniperus communis L. var. saxatilis Pall.;Kayalık ve taşlık alanlar, 17.05.2015, L7, O.Emin 22615\&Aksu, Php.

J. oxycedrus L. subsp. oxycedrus; Kayalık ve taşlık alanlar, 17.05.2015, L2, LC, O.Emin 22616\&Aksu, Php.

J. foetidissima Willd.; Kayalık ve taşlık alanlarda, 28.07.2015, L3, LC, O.Emin 22785\&Aksu, Php.

J. excelsa Bieb.; Kurak ve kayalık yamaçlarda, 28.07.2015, L4, O.Emin 22786\&Aksu, Medit., LC, Php.

\section{MAGNOLIOPHYTINA}

\section{RANUNCULACEAE}

Adonis aestivalis L.; Kayalık yamaç, 615 m, 17.05.2015, L1, O.Emin 22617\&Aksu, Hcrp.

Consolida orientalis (J. Gay) Schrodinger.; Yol kenarı, 17.05.2015, L1, O.Emin 22618\&Aksu, Php.

\section{Ranunculus repens $\mathrm{L}$.}

Nemli alan, 17.05.2015, L7, O.Emin 22619\&Aksu, Hcrp.

\section{BERBERIDACEAE}

Berberis integerrima Bunge.

Taşı yamaç, çalılık,17.05.2015, L1, O.Emin 22620\&Aksu, Php.

\section{PAPAVERACEAE}

Chelidonium majus L.; Yol kenarı, 02.04.2015, L2,

O.Emin 22580\&Aksu, Euro-Sib., Hcrp.

\section{Glaucium flavum Crantz}

Yol kenarı, 17.05.2015, L1, O.Emin 22621\&Aksu, Hcrp.

G. corniculatum (L.) Curtis.

Yol kenarı, 17.05.2015, L2, O.Emin 22622\&Aksu, Hcrp.

Papaver arenarium M. Bieb

Yamaç alan, 02.04.2015, L1, O.Emin 22581\&Aksu, Thp.

Fumaria officinalis $\mathrm{L}$.

Yol kenarı, 02.04.2015, L3, O.Emin 22582\&Aksu, Thp.

16. BRASSICACEAE 
Coluteocarpus vesicaria (L.) Holmboe

Taşlık yamaç, 17.05.2015, L7, O.Emin 22623\&Aksu, Ir-Tur, Hcrp.

Aethionema diastrophis Bunge

Yol kenarı, 17.05.2015, L1, O.Emin 22624\&Aksu, Hcrp.

A. armenum Boiss.; Kayalık yamaç, 17.05.2105, L7, O.Emin 22625\&Aksu, Ir-Tur, Hcrp.

Thlaspi perfoliatum L.; Nemli tarım alanları ve yol şevi, 17.05.2015, L5, O.Emin 22626\&Aksu, Cosm., Thp.

Tchihatchewia isatidea Boiss.; Yol kenarı, 17.05.2015, L6, O.Emin 22627\&Aksu, Endemik, VU, Ir-Tur, Hcrp.

Ricotia aucheri (Boiss.) B.L. Burtt.

Kayalık yamaç, 17.05.2015, L4, O.Emin 22628\&Aksu, IrTur, Hcrp.

Alyssum desertorum Stapf

Taşlık yamaç, 17.05.2015, L3, O.Emin 22629\&Aksu, Thp.

A. repens Baumg.

Çalıık yamaç, 28.07.2015, L3, O.Emin 22787\&Aksu, Hcrp.

A. murale Waldst. \& Kit.

Kayalık yamaç, 17.05.2015, L3, O.Emin 22630\&Aksu, Horp.

\section{Clypeola jonthlaspi L.}

Taşlık alan, 17.05.2015, L1, O.Emin 22631\&Aksu, Thp.

Draba hispida Willd.; Kayalık yamaç, 17.05.2015, L8, O.Emin 22632\&Aksu, Eux (mt)., Chp.

Arabis caucasica Vill.; Kayalık yamaç, 02.04.2015, L4, O.Emin 22583\&Aksu, Eux.(mt), Hcrp.

Cardamine raphanifolia Pourr. subsp. acris (Gris) O.E. Schulz

Su kenarı, 17.05.2015, L7, O.Emin 22633\&Aksu, Euro-Sib., Crp.

Alliaria petiolata (M. Bieb.) Cavara \& Grande

Yol kenarı, 17.05.2015, L3, O.Emin 22634\&Aksu, Thp.

Hesperis thyrsoidea Boiss.

Yol kenarı, 17.05.2015, L4, O.Emin 22635\&Aksu, Ir-Tur, Horp.

Erysimum uncinatifolium Boiss. \& A. Huet

Kumlu alan, 17.05.2015, L8, O.Emin 22636\&Aksu, Thp.

Sobolewskia clavata (Boiss.) Fenzl.

Kayalık yamaç, 17.05.2015, L3, O.Emin 22637\&Aksu, Hcrp.

Sisymbrium officinale (L.) Scop.

Yol kenarı, 17.05.2015, L2, O.Emin 22638\&Aksu, Thp.

17. CAPPARACEAE

Capparis sicula subsp. herbaceae (Willd.) Inocencio \& al. Yol kenarı, 17.05.2015, L3, O.Emin 22639\&Aksu, Chp.

18. CLEOMACEAE

\section{Cleome ornithopodioides $\mathrm{L}$.}

Yol kenarı, 28.07.2015, L2, O.Emin 22788\&Aksu, Medit., Thp.
19.RESEDACEAE

Reseda lutea $\mathrm{L}$.

Yol kenarı, 28.07.2015, L8, O.Emin 22789\&Aksu, Hcrp.

20.CISTACEAE

Helianthemum nummularium (L.) Mill. ; Eğimli taşlık kayalık alanlarda, 28.07.2015, L7, O.Emin 22790\&Aksu, Hcrp.

Fumana procumbens (Dunal) Gren. \& Godr.

Kayalık yamaç, 08.08.2014, L3, O.Emin 22383\&Aksu, Hcrp.

\section{VIOLACEAE}

Viola odorata L.; Gölgeli ve nemli meşçereler içerisinde, 02.04.2015, L3, O.Emin 22584\&Aksu, Hcrp.

V. sieheana Becker; Gölgeli ve su yakını alan, 17.05.2015, L3, O.Emin 22640\&Aksu, Hcrp.

V. tricolor $\mathrm{L}$.;

Su kenarı, 17.05.2015, L6, O.Emin 22641\&Aksu, Hcrp.

V. alba Besser

Yol kenarı, 17.05.2015, L4, O.Emin 22642\&Aksu, Hcrp.

22. POLYGALACEAE

Polygala anatolica Boiss. Et Heldr.; Yamaç, 17.05.2015, L4, O.Emin 22643\&Aksu,Cosm., Hcrp.

P. alpina (Dc.) Steud.; Çayır alan, 17.05.2015, L7, O.Emin 22644\&Aksu,Euro-Sib., Hcrp.

\section{CARYOPHYLLACEAE}

Cerastium armeniacum Gren.; Kayalık yamaç, 17.05.2015, L1, O.Emin 22645\&Aksu,Endemik, LC, Ir-Tur, Thp.

Dianthus floribundus Boiss.; Kayalık alanlar, 17.05.2015, L10, Ir-Tur.,O.Emin 22646\&Aksu, Hcrp.

D. crinitus $\mathrm{Sm}$. var. crinitus $\mathrm{Sm}$.

Kayalık yamaç, 17.05.2015, L13, O.Emin 22647\&Aksu, Horp.

D. erythrocoleus Boiss.; Kayalık yamaç, 28.07.2015, L8, O.Emin 22791\&Aksu, Endemik, NE, Ir-Tur., Hcrp.

D. artwinensis Schischk.; Yol kenarı, 28.07.2015, L12, O.Emin 22792\&Aksu, Endemik, LC, Ir-Tur., Hcrp.

Saponaria prostrata Willd.

Yol kenarı, 17.05.2015, L3, O.Emin 22648\&Aksu, Thp.

Vaccaria hispanica (Mill.) Rauschert

Tarla alanı, 17.05.2015, O.Emin 22649\&Aksu, L1, Thp.

Silene spergulifolia (Desf.) Bieb.; Çağıllık yamaç, 17.05.2015, L5, O.Emin 22650\&Aksu, Ir-Tur., Hcrp.

S. latifolia subsp. alba (Mill.) Greuter \& Burdet

Alpin vejetasyon, 08.08.2014, L8, O.Emin 22384\&Aksu, Horp.

Agrostemma githago L.

Tarla alanı, 17.05.2015, L1, O.Emin 22651\&Aksu, Thp.

Eremogone dianthoides (Sm.) Ikonn.; Yol kenarı, 17.05.2015, L4, O.Emin 22652\&Aksu, Ir-Tur., Hcrp. 


\section{POLYGONACEAE}

Polygonum carneum C. Koch.; Alpin vejetasyon, 08.08.2014, L9, O.Emin 22385\&Aksu, Eux. (mt). Hcrp.

$P$. aviculare $\mathrm{L}$.

Çorak alan, 08.08.2014, L3, O.Emin 22386\&Aksu, Cosm., Thp.

Rumex acetocella L.; Alpin vejetasyon, 08.08.2014, L9, O.Emin 22387\&Aksu, Cosm., Hcrp.

R. scutatus L.

Dere kenarı, 28.07.2015, L12, O.Emin 22793\&Aksu, Chp. R. alpinus $\mathrm{L}$.

Orman içi açıklık, 08.08.2014, L13, O.Emin 22388\&Aksu, Crp.

R. crispus L.; Çorak alan, 17.05.2015, L11, O.Emin 22653\&Aksu,

Cosm., Hcrp.

Atraphaxis billardieri subsp. tournefortii (Jaub. \& Spach) Lovelius; Kayalık yamaç, 08.08.2014, L11, O.Emin 22389\&Aksu, Ir-Tur., Hcrp.

\section{TAMARICACEAE}

Tamarix smyrnensis Bunge

Dere kenarı, 08.08.2014, L1, O.Emin 22390\&Aksu, Php.

Myricaria germanica (L.) Desv.

Dere kenarı, 17.05.2015, L4, O.Emin 22654\&Aksu, Php.

\section{HYPERICACEAE}

Hypericum scabrum L.; Yol kenarı taşlık alan, 17.05.2015, L3, O.Emin 22655\&Aksu, Ir-Tur., Hcrp.

\section{MALVACEAE}

Alcea calvertii (Boiss.) Boiss.; Taşlık yamaç, 28.07.2015, L1, O.Emin 22794\&Aksu, Endemik, LC, Ir-Tur., Hcrp.

28. LINACEAE

\section{Linum tenuifolium $\mathrm{L}$.}

Taşlık-çağıllık alan, 17.05.2015, L1, O.Emin 22656\&Aksu, Hcrp.

29. GERANIACEAE

Geranium lucidum L.

Yol kenarı, 17.05.2015, L3, O.Emin 22657\&Aksu, Thp.

G. purpureum Vill.

Ormanlık alan, 17.05.2015, L6, O.Emin 22658\&Aksu, Thp.

G. dissectum $\mathrm{L}$.

Yol kenarı, 17.05.2015, L3, O.Emin 22659\&Aksu, Thp.

G. collinum steph. ex. Willd.

Dere kenarı, 08.08.2014, L10, O.Emin 22391\&Aksu, IrTur., Crp.

G. ibericum Cav. subsp. ibericum; Alpin vejetasyon, 28.07.2015, L13, O.Emin 22795\&Aksu,Eux (mt)., Crp.

Erodium cicutarium (L.) L. Her. subsp. cicutarium Açık alan, 17.05.2015, L3, O.Emin 22660\&Aksu, Thp.

\section{ZYGOPHYLLACEAE}

Tribulus terrestris $\mathrm{L}$.
Açık ve kumlu alan, 28.07.2015, O.Emin 22796\&Aksu, L1, Thp.

\section{NITRARIACEAE}

Peganum harmala $\mathrm{L}$.

Yol kenarı, 17.05.2015, O.Emin 22661\&Aksu, L2, Hcrp.

32. RUTACEAE

Ruta suaveolens Dc.

Taşlık yamaç, 17.05.2015, L1, O.Emin 22662\&Aksu, LC, Hcrp.

33. SAPINDACEAE

Acer cappadocicum Gled. subsp. divergens (K. Koch ex Paxton) A.E. Murray; Yol kenarı, 28.07.2015, L3, O.Emin 22797\&Aksu,

Endemik, EN., Eux., Php.

\section{VITACEAE}

Vitis vinifera $L$. ;

Kültür alanı, 17.05.2015, L1, O.Emin 22663\&Aksu, Php.

\section{RHAMNACEAE}

Paliurus spina-christii Mill.; Yol ve dere kenarlarında, 02.04.2015, L1, O.Emin 22585\&Aksu, Chp.

Frangula alnus Mill. ; Dere kenarları, 08.08.2014, L5, O.Emin 22392\&Aksu, Euro-Sib., Php.

Rhamnus pallasii Fisch. \& Mey.

Kayalık yamaç, 28.07.2015, L3, O.Emin 22798\&Aksu, NE., Chp.

36. AQUIFOLIACEAE

Ilex colchica Pojark.; Ormanlık alan, 08.08.2014, L6, O.Emin 22393\&Aksu, Eux., Chp.

\section{ANACARDIACEAE}

Cotinus coggygria Scop.

Yol kenarı, 17.05.2015, L1, O.Emin 22664\&Aksu, Medit.,Chp.

Rhus coriaria $\mathrm{L}$.

Yol kenarı, 08.08.2014, L3, O.Emin 22394\&Aksu, Medit., Chp.

\section{FABACEAE}

Sophora alopecuroides $\mathrm{L}$.

Tarla kenarı, 17.05.2015, L1, O.Emin 22665\&Aksu, Crp.

Cytisus hirsutus L.

Yol kenarı, 08.08.2014, L2, O.Emin 22395\&Aksu, Chp.

Genista aucheri Boiss. ; Yol kenarı, taşlı yamaç, 17.05.2015, L2, O.Emin 22666\&Aksu, Endemik, LC, Ir-Tur, Chp.

Robinia pseudoacacia L.; Yol kenarı, 08.08.2014, L3, O.Emin 22396\&Aksu, Egzotik, Doğallaşmış, Php.

Colutea armena Boiss. \& A. Huet

Kayalık yamaç, 28.07.2015, L2, O.Emin 22799\&Aksu, Php. Caragana grandiflora (M. Bieb.) DC.

Kayalık yamaç, 08.08.2014, L3, O.Emin 22397\&Aksu, IrTur, Chp. 
Chesneya elegans Fomin. ; Tepe etekleri, 17.05.2015, L2, O.Emin 22667\&Aksu, Endemik, LC, Ir-Tur, Hcrp.

Astragalus campylosema Boiss.; Taşlık-kayalık yamaç, 17.05.2015, L3, O.Emin 22668 \&Aksu, Endemik, NE, IrTur, Hcrp.

A. xylobasis Freyn et Bornm.. ; Çayırlık, 17.05.2015, L5, O.Emin 22669\&Aksu, Endemik, NE, Ir-Tur, Hcrp.

A. taochius Woronov; Çayırlık, 17.05.2015, L1, O.Emin 22670\&Aksu, Hcrp.

Astracantha microcephala (Willd.) Podlech

Yol kenarı, 28.07.2015, L3, O.Emin 22800\&Aksu, Hcrp.

Vicia cracca L.;Yol kenarı, 17.05.2015, L11, O.Emin 22671\&Aksu, Euro-Sib, Hcrp.

V. peregrina L.; Quercus petraea subsp. iberica çalılıkları, 17.05.2015, L3, O.Emin 22672\&Aksu, Thp.

V. sativa subsp. nigra (L.) Ehrh.; Çayırlık alan, 17.05.2015, L10, O.Emin 22673\&Aksu, Cosm., Thp.

Lathyrus digitatus (M. Bieb.) Fiori.; Yol kenarı, çayırlık alan, 28.07.2015, L8, O.Emin 22801\&Aksu, Hcrp.

L. woronowii Bornm.

Çağıllık alan, 17.05.2015, L2, O.Emin 22674\&Aksu,

Endemik, CR, Eux., Thp.

Pisum sativum $\mathrm{L}$.

Yol kenarı, 17.05.2015, L1, O.Emin 22675\&Aksu, Thp.

Trifolium repens $\mathrm{L}$.

Otlak alan, 08.08.2014, L9, O.Emin 22398\&Aksu, Chp.

T. ambiguum M. Bieb.

Alpin vejetasyon, 08.08.2014, L8, O.Emin 22399\&Aksu, Horp.

T. hybridum L. var. hybridum

Çayırlık alan, 08.08.2014, L6, O.Emin 22400\&Aksu, Hcrp.

T. campestre Schreb.

Kuru yamaç, 17.05.2015, L3, O.Emin 22676\&Aksu, Thp.

T. pratense $\mathrm{L}$.

Çayırlık alan, 17.05.2015, L8, O.Emin 22677\&Aksu, Hcrp.

T. sylvaticum Gerard ex Loisel

Açık alan, 1592 m, 28.07.2015, L5, O.Emin 22802\&Aksu,

Thp.

T. arvense $\mathrm{L}$.

Taşlık alan, 28.07.2015, L4, O.Emin 22803\&Aksu, Thp.

Medicago lupulina $\mathrm{L}$.

Yol kenarı, tarla alanı, 17.05.2015, L3, O.Emin 22678\&Aksu,

Cosm., Hcrp.

\section{M. sativa L.}

Taşlık yamaç, 08.08.2014, L4, O.Emin 22402\&Aksu,

Cosm., Hcrp.

Lotus tenuis Waldst. \& Kit.

Dere kenarı, 28.07.2015, L1, O.Emin 22804\&Aksu, Hcrp.

Securigera orientalis (Mill.) Lassen.

Taşlık alan, 17.05.2015, L1, O.Emin 22679\&Aksu, Chp.
Hedysarum huetii Boiss.; Yol kenarı taşılık yamaç, 17.05.2015, L1, O.Emin 22680\&Aksu, Ir-Tur, Hcrp.

Onobrychis huetiana Boiss.;Yol kenarı, 17.05.2015, L1, O.Emin 22681\&Aksu, Endemik, NT, Ir-Tur, Hcrp.

O. stenostachya Freyn; Yol kenarı, 17.05.2015, L6, O.Emin 22682\&Aksu, Endemik, NE, Ir-Tur, Hcrp.

39. ROSACEAE

Alchemilla caucasica Buser

Alpin vejetasyon, 08.08.2014, L9, O.Emin 22403\&Aksu, Eux. (mt)., Crp.

A. mollis (Buser) Rothm.

Dere kenarı, 08.08.2014, L6, O.Emin 22404\&Aksu, Crp.

Cotoneaster morulus Pojark.

Taşık yamaç, 17.05.2015, L1, O.Emin 22683\&Aksu, LC, Chp.

Crataegus monogyna Jacq.

Orman içi açıklık, 08.08.2014, L12, O.Emin 22405\&Aksu, Php.

C. orientalis Pallas ex Bieb. var. orientalis

Kayalık yer, orman alanı, 08.08.2014, L12, O.Emin 22406\&Aksu, Php.

Fragaria vesca $\mathrm{L}$.

Orman içi, 28.07.2015, L13, O.Emin 22805\&Aksu, Hcrp.

Geum urbanum L.;Yol kenarı, 28.07.215, L8, O.Emin 22806\&Aksu, Euro-Sib., Crp.

Malus sylvestris Miller. subsp. orientalis (A. Uglitzkich) Browicz var. orientalis

Orman içi açıklık, 08.08.2014, L6, O.Emin 22407\&Aksu, Php.

Potentilla recta $\mathrm{L}$.

Yol kenarı, 17.05.2015, L2, O.Emin 22684\&Aksu, Crp.

P.erecta (L.) Rausch

Açık ormanlık alan, 08.08.2014, L6, O.Emin 22408\&Aksu, Crp.

$P$. reptans $L$.

Dere kenarı, 08.08.2014, L7, O.Emin 22409\&Aksu, Hcrp.

Prunus cerasus $\mathrm{L}$.

Seyrek orman, dik yamaç, 08.08.2014, L5, O.Emin 22410\&Aksu, Php.

Pyrus elaeagnifolia Pallas subsp. elaeagnifolia

Yol kenarı, 28.07.2015, L3, O.Emin 22807\&Aksu, Php.

Rosa canina L.

Açık alan, 28.07.2015, L8, O.Emin 22808\&Aksu, Chp.

R. xanthina Lindl.

Kuru yamaç, 28.07.2015, L3, O.Emin 22809\&Aksu, Chp.

Rubus idaeus L.;Orman içi açık alan, 28.07.2015, L3, O.Emin 22810\&Aksu, Chp.

R. sp.

Yol kenarı, 28.07.2015, L3, O.Emin 22811\&Aksu, Chp.

Sorbus aucuparia L.; Orman içi açıklık, 08.08.2014, L10, O.Emin 22411\&Aksu, Euro-Sib., Php. 
S. caucasica Zinserl var. caucasica;Açık alan, 08.08.2014, L12, O.Emin 22412\&Aksu, Euro-Sib., Php.

S. subfusca (Ledeb.) Boiss.; Subalpin çayırlık, 08.08.2014, L7, O.Emin 22413\&Aksu, LC, Eux., Php.

S. umbellata (Desf.) Fritsch.

Açık alan, 08.08.2014, L12, O.Emin 22414\&Aksu, Php.

40. LYTHRACEAE

Punica granatum $\mathrm{L}$.

Yol kenarı, 08.08.2014, L1, O.Emin 22415\&Aksu, Php.

41. ONAGRACEAE

Epilobium angustifolium $\mathrm{L}$.

Kayalık yamaç, 08.08.2014, L8, O.Emin 22416\&Aksu, Crp.

E. roseum Schreber subsp. subsessile (Boiss.) P.H. Raven.

Dere kenarı, 17.05.2015, L2, O.Emin 22685\&Aksu, Crp.

E. ponticum Hausskn.

Alpin vejetasyon, 08.08.2014, L9, O.Emin 22417\&Aksu,

Crp.

\section{CRASSULACEAE}

Sedum album $\mathrm{L}$.

Kayalık yamaç, kaya yarıkları, 28.07.2015, L8, O.Emin 22812\&Aksu, Crp.

S. stoloniferum S. G. Gmel.

Kayalık yamaç, 28.07.2015, L7, O.Emin 22813\&Aksu,

Eux., Hcrp.

Sempervivum minus Turril ex Wale

Kayalık yamaç, 28.07.2015, L13, O.Emin 22814\&Aksu,

LC, Horp.

\section{SAXIFRAGACEAE}

Saxifraga paniculata Mill. subsp. paniculata

Kaya yarıkları, 28.07.2015, L12, O.Emin 22815\&Aksu,

Euro-Sib., Chp.

\section{GROSSULARIACEAE}

Ribes alpinum $\mathrm{L}$.

Orman içi açıklık, 08.08.2014, L6, O.Emin 22418\&Aksu,

O.Emin 22866\&Aksu, Chp.

\section{APIACEAE}

\section{Sanicula europaea $\mathrm{L}$.}

Orman içi gölgelik alan, 08.08.2014, L5, O.Emin 22419\&Aksu, Euro-Sib., Hcrp.

Astrantia maxima Pall. subsp. maxima

Çayırlık alan, 1971 m, 08.08.2014, L12, O.Emin 22420\&Aksu, NT, Eux., Hcrp.

Eryngium caeruleum M. Bieb.; Kuru alan, 08.08.2014, L1, O.Emin 22421\&Aksu,Ir-Tur., Hcrp.

\section{Chaerophyllum bulbosum $\mathrm{L}$.}

Tarla kenarı, 08.08.2014, L4, O.Emin 22422\&Aksu, Euro-

Sib.,Thp.

C. macrospermum (Spreng.) Fisch. \& C. A. Mey.

Taşlık alan, 17.05.2015, L4, O.Emin 22686\&Aksu, Ir-Tur., Horp.

Carum carvi L.
Nemli çayır, 08.08.2014, L9, O.Emin 22423\&Aksu, Chp.

Chamaesciadium acaule (Bieb.) Boiss.; Kayalık alan, 17.05.2015, L8, O.Emin 22687\&Aksu, Hyrc-Eux., Hcrp.

Pimpinella affinis Ledeb.;Kayalık ve taşlık alan, 08.08.2014, L8, O.Emin 22424\&Aksu, Hcrp.

Foeniculum vulgare Mill.

Yol kenarı, 08.08.2014, L2, O.Emin 22425\&Aksu, Hcrp.

Bupleurum schistosum Woronow; Kayalık ve kuru yamaç, 17.05.2015, L3, O.Emin 22688\&Aksu, Endemik, EN, Ir-Tur, Hcrp.

Heracleum sphondylium L. subsp. cyclocarpum (C. Koch) Davis

Dere kenarı, orman içi, 08.08.2014, L10, O.Emin 22426\&Aksu, LC, Eux., Hcrp.

H. apiifolium Boiss.; Dere kanarı, çayırlık alan, 17.05.2015, L13, O.Emin 22689\&Aksu, NE, Eux (mt)., Hcrp.

Zosima absinthifolia (Vent.) Link

Taşlı yamaç, 17.05.2015, L12, O.Emin 22690\&Aksu, Hcrp.

Torilis japonica (Houtt.) Dc.; Kayalık yamaç, çayırlık alan, 17.05.2015, L3, O.Emin 22691\&Aksu, Thp.

Caucalis platycarpos $\mathrm{L}$.

Yol kenarı, 08.08.2014, L13, O.Emin 22427\&Aksu, Thp.

Daucus carota L.

Yol kenarı, 17.05.2015, L1, O.Emin 22692\&Aksu, Hcrp.

Aethusa cynapium L.

Yol kenarı, 17.05.2015, L1, O.Emin 22693\&Aksu, Euro-Sib. Thp.

Anthriscus caucalis Bieb.

Yol kenarı, 17.05.2015, L5, O.Emin 22694\&Aksu, Thp.

46.ARALIACEAE

Hedera helix L.; Orman içi, ağaçlar üzerine tırmanıcı, 08.08.2014, L12, O.Emin 22428\&Aksu, Chp.

H. colchica (C.Koch.) C.Koch

Orman içi, 08.08.2014, L6, O.Emin 22429\&Aksu, Eux., Chp.

47. CORNACEAE

Cornus sanguinea L. subsp. cilicica (Wangerin) Chamberlain.

Ormanlık alan, 08.08.2014, L4, O.Emin 22430\&Aksu, E. Medit., Php.

C. mas $\mathrm{L}$.

Açık alan, 08.08.2014, L4, O.Emin 22431\&Aksu, Euro-Sib., Php.

48. ADOXACEAE

Sambucus nigra $\mathrm{L}$.

Orman içi, 08.08.2014, L6, O.Emin 22432\&Aksu, EuroSib., Php.

Viburnum opulus L.; Orman kenarı, 08.08.2014, L6, O.Emin 22433\&Aksu, Euro-Sib., Chp.

V. orientale Pallas 
Orman içi, 08.08.2014, L6, O.Emin 22434\&Aksu, Eux., Chp.

49. CAPRIFOLIACEAE

Lonicera caucasica Pall.

Yol kenarı, 28.07.2015, L3, O.Emin 22816\&Aksu, Chp.

Centranthus longiflorus Stev. ; Kayalık yamaç, yol kenarı, 17.05.2015, L2, O.Emin 22695\&Aksu, Ir-Tur., Crp.

Valeriana alliariifolia Adams; Subalpin vejetasyon, dere kenarı, 28.07.2015, L7, O.Emin 22817\&Aksu, Hcrp.

Cephalaria aristata K. Koch.

Çayırlık alan, 28.07.2015, L3, O.Emin 22818\&Aksu, Thp.

Dipsacus pilosus L.

Yol kenarı, 08.08.2014, L4, O.Emin 22435\&Aksu, EuroSib., Thp.

Knautia montana (Bieb.) DC.; Yol kenarı, 08.08.2014, L3, O.Emin 22436\&Aksu, EN, Euro-Sib., Thp.

Scabiosa columbaria L. ; Kayalık ve taşlık yamaç, 08.08.2014, L7, O.Emin 22437\&Aksu, Crp.

S. crinita Kotschy Et Boiss.;Çayırlık kenarı, 08.08.2014, L13, O.Emin 22438\&Aksu, Ir-Tur. Crp.

Morina persica L.; Çakıllık alan, 28.07.2015, L5, O.Emin 22819\&Aksu,Endemik, EN, Ir-Tur. Hcrp.

\section{ASTERACEAE}

\section{Xanthium strumarium $\mathrm{L}$.}

Yol kenarı, 08.08.2014, L3, O.Emin 22439\&Aksu, Thp.

Telekia speciosa (Schreb.) Baumg.; Dere kenarı, 08.08.2014, L5, O.Emin 22440\&Aksu, Euro-Sib., Hcrp.

Inula orientalis Lam.; Açık yamaç, 08.08.2014, L10, O.Emin 22441\&Aksu,Eux (mt), Crp.

Antennaria dioica (L.) Gaertn.; Taşlık yamaç, 28.07.2015, L7, O.Emin 22820\&Aksu, Euro-Sib. Hcrp.

Helichrysum armenium DC. ; Kayalık yamaç, 17.05.2015, L11, O.Emin 22696\&Aksu, Ir-Tur., Hcrp.

H. orientale (L.) Vaill.

Açık alan, 28.07.2015, L12, O.Emin 22821\&Aksu, Medit., Hcrp.

Gnaphalium sylvaticum L.; Alpin vejetasyon, 08.08.2014, L9, O.Emin 22442\&Aksu,Euro-Sib., Crp.

Solidago virgaurea L. subsp. alpestris (Waldst. \& Kit.) Gremli

Yol kenarı, 08.08.2014, L8, O.Emin 22443\&Aksu, EuroSib.,Hcrp.

Kemulariella caucasica (Willd.) Tamamsch.; Kayalık yamaç, 08.08.2014, L13, O.Emin 22444\&Aksu,VU, Eux., Chp.

Erigeron caucasicus Stev.

Kayalık yamaç, 28.07.2015, L13, O.Emin 22822\&Aksu, Horp.

E. acer L.; Yol kenarı, 28.07.2015, L12, O.Emin 22823\&Aksu,Euro-Sib., Hcrp.
Bellis perennis L.; Açık alan, 08.08.2014, L4, O.Emin 22445\&Aksu,Euro-Sib., Hcrp.

Doronicum orientale Hoffm.

Ormanlık alan, 17.05.2015, L12, O.Emin 22697\&Aksu, Crp.

Senecio pseudoorientalis Schischk.; Çayırlık yamaç, 17.05.2015, L9, O.Emin 22698\&Aksu, Ir-Tur., Hcrp.

Tussilago farfara L.; Nemli alan, 17.05.2015, L7, O.Emin 22699\&Aksu, Euro-Sib., Crp.

Petasites albus (L.) Gaertner; Dere kenarı, taşlık alan, 08.08.2014, L7, O.Emin 22446\&Aksu, Euro-Sib. Crp.

Eupatorium cannabinum $\mathrm{L}$.

Yol kenarı, 08.08.2014, L3, O.Emin 22447\&Aksu, Euro-Sib. Hcrp.

Cota tinctoria (L.) J. Gay

Yol kenarı, 08.08.2014, L10, O.Emin 22448\&Aksu, Cosm., Horp.

Achillea arabica Kotschy; Kayalık yamaç, 17.05.2015, L3, O.Emin 22700\&Aksu, Ir-Tur. Hcrp.

Tanacetum macrophyllum (Waldst. \& Kit.) Schultz Bip.; Dere kenarı, 08.08.2014, L7, O.Emin 22449\&Aksu, EuroSib, Hcrp.

T. parthenium (L.) Schultz Bip.; Dere kenarı, 08.08.2014, L10, O.Emin 22450\&Aksu, Cosm., Hcrp.

T. argenteum ( Lam.) Wild. subsp. canum (K.Koch) Grierson var. canum; Çağıllık yamaç, taş yarıkları, 08.08.2014, L4, O.Emin 22451\&Aksu, Hcrp.

Tripleurospermum melanolepis (Boiss. et Buhse) Pobed. Çağıllık alan, 17.05.2015, L7, O.Emin 22701\&Aksu, Crp.

T. caucasicum (Willd.) Hayek.

Çağıllık alan, 17.05.2015, L12, O.Emin 22702\&Aksu, Crp.

Onopordum acanthium L.

Kayalık yamaç, 08.08.2014, L10, O.Emin 22452\&Aksu, Horp.

Cirsium caucasicum (Adams) Petrak; Alpin vejetasyon, 08.08.2014, L9, O.Emin 22453\&Aksu,VU, Eux (mt)., Hcrp.

C. simplex C. A. Mey. subsp. armenum (DC.) Petrak

Dere kenarı, 08.08.2014, L13, O.Emin 22454\&Aksu, NE, Crp.

C. arvense (L.) Scop.; Ormanlık alan, 28.07.2015, L5, O.Emin 22824\&Aksu, Cosm., Hcrp.

C. pubigerum (Desf.) DC.

Yol kenarı, 28.07.2015, L3, O.Emin 22825\&Aksu, Hcrp.

Carduus hamulosus Ehrh. subsp. hamulosus; Çayırlık alan, 08.08.2014, L10, O.Emin 22455\&Aksu, Euro-Sib., Crp.

Jurinea moschus subsp. pinnatisecta (Boiss.) Greuter Taşlık alan, 08.08.2014, L9, O.Emin 22456\&Aksu, Ir-Tur. Hcrp.

Centaurea virgata Lam.; Taşlık yamaç, 28.07.2015, L3, O.Emin 22826\&Aksu, Ir-Tur., Hcrp. 
C. urvillei DC. subsp. stepposa Wagenitz; Yol kenarı, taşlık yamaç, 17.05.2015, L1, O.Emin 22703\&Aksu, Endemik, NE, Ir-Tur., Hcrp.

C. pseudoscabiosa Boiss. \& Buhse

Açık alan, 28.07.2015, L12, O.Emin 22827\&Aksu, Ir-Tur., Hcrp.

Cyanus triumfetti (All.) Dostal ex A. Love \& D. Love

Yol kenarı, 17.05.2015, L6, O.Emin 22704\&Aksu, Cosm., Horp.

C. segetum Hill.; Kayalık ve taşlık yamaç, 28.07.2015, L8, O.Emin 22828\&Aksu, Hcrp.

Psephellus dealbata Willd.

Yol kenarı, 28.07.2015, L12, O.Emin 22829\&Aksu, Hcrp.

P. pulcherrimus (Willd.) Wagenitz

Kayalık yamaç, 08.08.2014, L9, O.Emin 22457\&Aksu, Chp.

P. simplicicaulis (Boiss. \& A. Huet) Wagenitz

Kaya yarıkları, 17.05.2015, L10, O.Emin 22705\&Aksu, Eux, Horp.

P. taochius Sosn.; Taşlık alan, 17.05.2015, L1, O.Emin 22706\&Aksu, Endemik, VU, Ir-Tur. Hcrp.

P. pecho (Albov) Wagenitz; Kayalık yamaç, 28.07.2015, L3, O.Emin 22830\&Aksu, Endemik, EN, Eux., Hcrp.

\section{Carthamus lanatus $\mathrm{L}$.}

Kurak yamaç, 17.05.2015, L2, O.Emin 22707\&Aksu, Hcrp.

Xeranthemum annuum L.; Taşlık-kayalık alan, 08.08.2014, L3, O.Emin 22458\&Aksu, Ir-Tur., Cosm., Thp.

Echinops sphaerocephalus L.; Taşlık yamaç, 28.07.2015, L3, O.Emin 22831\&Aksu, Euro-Sib., Hcrp.

Uechtritzia armena Freyn et Sint. ex Freyn; Açık alan, 28.07.2015, L3, O.Emin 22832\&Aksu,Endemik, EN, Ir-Tur. Hcrp.

Scorzonera seidlidzii Boiss.

Taşlık yamaç, 28.07.2015, L13, O.Emin 22833\&Aksu, Horp.

S. dzhawakhetica Sosn. ex Grossh.; Yol kenarı, 28.07.2015, L2, O.Emin 22834\&Aksu, Endemik, VU., Hcrp.

S. ketzkhovelii Sosn. ex Grossh.

Yol kenarı, 28.07.2015, L12, O.Emin 22835\&Aksu, Hcrp.

Cichorium intybus L.; Yol kenarı, çayırlık alan, 08.08.2014, L4, O.Emin 22459\&Aksu, Cosm., Hcrp.

Tragopogon reticulatus Boiss. \& Huet

Taşlık yamaç, 02.04.2015, L2, O.Emin 22586\&Aksu, Hcrp. Leontodon hispidus L. ; Taşlık ve kayalık yamaç, 08.08.2014, L1, O.Emin 22460\&Aksu, Euro-Sib., Crp.

\section{Sonchus oleraceus $\mathrm{L}$.}

Tarla kenarı, 17.05.2015, L1, O.Emin 22708\&Aksu, Hcrp.

S. arvensis $\mathrm{L}$.

Yol kenarı, 17.05.2015, L2, O.Emin 22709\&Aksu, Hcrp.

Reichardia glauca Matthews
Yol kenarı, 28.07.2015, L11, O.Emin 22836\&Aksu, Ir-Tur., Horp.

Hieracium pannosum Boiss.

Taşlık alan, 28.07.2015, L7, O.Emin 22837\&Aksu, Medit. Hcrp.

Pilosella hoppeana (Schultes) C. H. \& F. W. ; Schultz Taşlık yamaç, 28.07.2015, L13, O.Emin 22838\&Aksu, Chp.

Lactuca serriola L. ; Kayalık yamaç, 08.08.2014, L6, O.Emin 22461\&Aksu, Euro-Sib., Hcrp.

Taraxacum turcicum Soest; Çorak alan, 28.07.2015, L4, O.Emin 22839\&Aksu,Endemik, LC, Medit., Hcrp.

Crepis foetida L. subsp. rhoeadifolia (M. Bieb.) Celak. Kayalık yamaç, 08.08.2014, L3, O.Emin 22462\&Aksu, Cosm., Thp.

Lapsana communis $\mathrm{L}$.

Yol kenarı, 02.04.2015, L1, O.Emin 22587\&Aksu, Hcrp.

51. CAMPANULACEAE

\section{Campanula sibirica L.}

Kayalık yamaç, 02.04.2015, L1, O.Emin 22588\&Aksu, Thp. C. glomerata L. subsp. hidpida (Witasek) hayek; Açık ormanlık alan, 08.08.2014, L10, O.Emin 22463\&Aksu, Euro-Sib., Hcrp.

C. alliarifolia Willd.; Picea orientalis ormanı, 08.08.2014, L6, O.Emin 22464\&Aksu, Eux., Hcrp.

C. saxifraga subsp. aucheri (A.DC.) Ogan.; Alpin vejetasyon, 08.08.2014, L9, O.Emin 22465\&Aksu, Eux (mt)., Crp.

C. saxonorum Gand.; Çağıllık alan, 17.05.2015, L1, O.Emin 22710\&Aksu, Endemik, NE, Ir-Tur., Thp.

C. olympica Boiss. ; Kayalık yamaç, 02.04.2015, L1, O.Emin 22589\&Aksu, Eux., Hcrp.

Asyneuma amplexicaule (Willd.) Hand-Mazz.

Kayalık yamaç, 08.08.2014, L9, O.Emin 22466\&Aksu, Horp.

52. ERICACEAE

Rhododendron luteum Sweet; Yapraklı ve iğne yapraklı türlerle bir arada, 17.05.2015, L6, O.Emin 22711\&Aksu, Eux., Chp.

R. ponticum L. ; Orman içi açıklık, 08.08.2014, L10, O.Emin 22467\&Aksu,Eux., Php.

Vaccinium myrtillus L.; Alpin vejetasyon, kayalık yamaç, 08.08.2014, L13, O.Emin 22468\&Aksu, Euro-Sib., Chp.

V. arctostaphyllos L.; Picea orientalis (L.) Link ormanı, 08.08.2014, L6, O.Emin 22469\&Aksu, Eux., Chp.

Orthilia secunda (L.) House; Picea orientalis (L.) Link ormanı altı, 08.08.2014, L6, O.Emin 22470\&Aksu, Crp.

Monotropa hypopithys L.; Picea orientalis (L.) Link ormanı altı, 08.08.2014, L10, O.Emin 22471\&Aksu, Thp.

\section{PRIMULACEAE}

Primula veris L.; Çayır alan, 02.04.2015, L3, O.Emin 22590\&Aksu, Euro-Sib., Hcrp. 
P. auriculata Lam.; Dere kenarı, 17.05.2015, L7, O.Emin 22712\&Aksu,LC, Ir-Tur., Hcrp.

Androsace armeniaca var. macrantha (Boiss. \& A. Huet) Martelli

Kayalık yamaç, 17.05.2015, L11, O.Emin 22713\&Aksu, Endemik, LC, Ir-Tur, Hcrp.

Cyclamen coum Mill.

Kayalık yamaç, 02.04.2015, L10, O.Emin 22591\&Aksu, Crp.

Lysimachia punctata L.; Dere kenarı, nemli-gölgeli alan, 08.08.2014, L7, O.Emin 22472\&Aksu, Hyrc-Eux., Hcrp.

Anagallis arvensis $\mathrm{L}$. var. arvensis

Kayalık yamaç, 17.05.2015, L3, O.Emin 22714\&Aksu, Thp.

54. EBENACEAE

Diospyros lotus L.

Yol kenarı, 08.08.2014, L3, O.Emin 22473\&Aksu, Php.

55. OLEACEAE

Jasminum fruticans $\mathrm{L}$.

Açık alan, 17.05.2015, L2, O.Emin 22715\&Aksu, Medit.

Crp.

Fraxinus angustifolia Valh. subsp. oxycarpa (Bieb. Ex Wild) Franco \& Rocha; Yol kenarı, 08.08.2014, L5, O.Emin 22474\&Aksu, Euro-Sib., Php.

Olea europaea L. ; Kayalık yamaç, 06.08.2014, L3, O.Emin 22475\&Aksu, Medit., Php.

\section{APOCYNACEAE}

Vinca herbacea Waldst. \& Kit.

Çalılık alan, 17.05.2015, L3, O.Emin 22716\&Aksu, Chp.

Vincetoxicum scandens Somm. \& Lev.

Ormanlık alan, 08.08.2014, L10, O.Emin 22476\&Aksu, Horp.

Apocynum venetum $\mathrm{L}$.

Yol kenarı, 28.07.2015, L2, O.Emin 22840\&Aksu, Medit. Horp.

Periploca graeca L. var. graeca Boiss. \& Heldr.; Dere kenarı, 02.04.2015, L2, O.Emin 22592\&Aksu, E. Medit., Chp.

\section{GENTIANACEAE}

Centaurium erythraea Rafn. subsp. erythraea; Güneşli bakılar ve kayalık alan, 28.07.2015, L1, O.Emin 22841\&Aksu, LC, Euro-Sib., Crp.

Gentiana septemfida Pall.; Alpin vejetasyon, 08.08.2014, L9, O.Emin 22477\&Aksu, Hyrc-Eux. (mt), Hcrp.

G. verna L. subsp. pontica (Soltok.) Hayek; Alpin vejetasyon, 08.08.2014, L9, O.Emin 22478\&Aksu, HyrcEux. (mt), Hcrp.

G. asclepiadea L.; Orman içi açıklık alan, 08.08.2014, L6, O.Emin 22479\&Aksu, Euro-Sib. Hcrp.

Gentianopsis ciliata subsp. blepharophora (Bordz.); Subalpin vejetasyon, 08.08.2014, L12, O.Emin 22480\&Aksu, Hyrc-Eux. (mt), Hcrp.
Swertia iberica Fisch. ex C.A.Mey.; Alpin vejetasyon, 08.08.2014, L9, O.Emin 22481\&Aksu, LC, Eux (mt)., Hcrp.

\section{CONVOLVULACEAE}

Convolvulus pseudoscammonia C. Koch; Yol kenarı, 17.05.2015, L1, O.Emin 22717\&Aksu, Endemik, LC, Ir-Tur. Horp.

C. arvensis L.; Açıklık alan, taşlık-kayalık alanlarda, 17.05.2015, L1, O.Emin 22718\&Aksu, Cosm., Hcrp.

C. Ianatus Vahl.; Çağıllık alan, 17.05.2015, L2, O.Emin 22719\&Aksu, Hcrp.

Calystegia silvatica (Kit.) Griseb.

Yol kenarı, 08.08.2014, L5, O.Emin 22482\&Aksu, Hcrp.

Cuscuta monogyna Vahl

Çalılar üzerinde, 28.07.2015, L1, O.Emin 22842\&Aksu, Hcrp.

59. BORAGINACEAE

Heliotropium ellipticum Ledeb.; Kuru taşlı yamaç, 08.08.2014, L2, O.Emin 22483\&Aksu, Ir-Tur., Thp.

Myosotis sylvatica Ehr. ex Hoffm. ; Subalpin vejetasyon, 17.05.2015, L8, O.Emin 22720\&Aksu, Hcrp.

M. heteropodo Trautv.

Kayalık yamaç, 17.05.2015, L7, O.Emin 22721\&Aksu, Crp. Paracaryum sp.

Taşlık yamaç, 17.05.2015, L1, O.Emin 22722\&Aksu, Hcrp. Cynoglossum creticum Mill.

Alpin vejetasyon, 17.05.2015, L9, O.Emin 22723\&Aksu, Hcrp.

Buglossoides arvensis (L.) Johnston

Taşlık alan, 17.05.2015, L6, O.Emin 22724\&Aksu, Thp.

Echium italicum L.

Taşlı yamaç, 17.05.2015, L3, O.Emin 22725\&Aksu, Medit., Crp.

E. vulgare $\mathrm{L}$.

Yol kenarı, 28.07.2015, L3, O.Emin 22843\&Aksu, EuroSib., Crp.

Onosma tenuiflora Willd.

Yol kenarı, 17.05.2015, L1, O.Emin 22726\&Aksu, Ir-Tur. Horp.

O. nigricaulis Riedl; Yol kenarı, taşlık yamaç, 17.05.2015, L3, O.Emin 22727\&Aksu, Hcrp.

O. sericeum Willd.

Taşlık alan, 17.05.2015, L1, O.Emin 22728\&Aksu, Ir-Tur. Hcrp.

Cerinthe minor L. ; Kuru yamaç, 08.08.2014, L12, O.Emin 22484\&Aksu, Euro-Sib., Hcrp.

Symphytum asperum Lepechin; Çayırlık alan, 08.08.2014, L7, O.Emin 22485\&Aksu, Hyrc-Eux. Hcrp.

Anchusa leptophylla Roem. \& Schult. Yol kenarı, 17.05.2015, L4, O.Emin 22729\&Aksu, Hcrp. Nonea obtusifolia (Willd.) DC.; Tarla kenarı, 17.05.2015, L1, O.Emin 22730\&Aksu, Medit., Thp. 
N. versicolor (Steven) Sweet; Taşlık yamaç, 17.05.2015, L1, O.Emin 22731\&Aksu, Euro-Sib., Thp.

N. caspica (Willd.) G. Don

Yol kenarı, 02.04.2015, L1, O.Emin 22593\&Aksu, Ir-Tur. Thp.

Lithospermum officinale $L$.

Yol kenarı, 02.04.2015, L1, O.Emin 22594\&Aksu, Euro-Sib. Horp.

Moltkia aurea Boiss.; Kayalık alan, 17.05.2015, L2, O.Emin 22732\&Aksu, Endemik, LC, Ir-Tur. Hcrp.

Alkanna cordifolia C. Koch; Taşlık alan, 17.05.2015, L4, O.Emin 22733\&Aksu, Endemik, LC, Medit. Hcrp.

60. SOLANACEAE

Solanum americanum Mill.; Dere kenarı, 08.08.2014, L5, O.Emin 22486\&Aksu, Cosm., Thp.

Atropa belladonna L. ; Yol kenarı, gölgelik alan, 08.08.2014, L6,

O.Emin 22487\&Aksu, Euro-Sib., Hcrp.

Datura stramonium L.; Tarla yanı, 08.08.2014, L3, O.Emin 22488\&Aksu, Cosm., Thp.

Hyoscyamus niger L. ; Yol kenarı, 17.05.2015, L3, O.Emin 22734\&Aksu, Hcrp.

\section{SCROPHULARIACEAE}

Scrophularia ilwensis K. Koch; Yol kenarı, 17.05.2015, L1, O.Emin 22735\&Aksu, Ir-Tur., Thp.

Verbascum cheiranthifolium Boiss.; Yol kenarı, 28.07.2015, L12, O.Emin 22844\&Aksu, Hcrp.

V. orientale (L.) All.; Yol kenarı, 17.05.2015, L1, O.Emin 22736\&Aksu, Medit., Thp.

\section{OROBANCHACEAE}

Pedicularis wilhelmsiana Fischer Ex Bieb.; Subalpin vejetasyon, 08.08.2014, L12, O.Emin 22489\&Aksu, NE, Eux. (mt)., Hcrp.

P. pontica Boiss.; Alpin vejetasyon, 08.08.2014, L9, O.Emin 22490\&Aksu, NE, Eux. (mt)., Hcrp.

Rhinanthus serotinus subsp. aestivalis (N.W.Zinger) Dostal

Orman kenarı, 08.08.2014, L6, O.Emin 22491\&Aksu, Thp. Rhynchocorys elephas (L.) Griseb. subsp. elephas; Çayırlık alan, 08.08.2014, L7, O.Emin 22492\&Aksu, Euro-Sib., Horp.

R. stricta (C.Koch) Albov; Kayalık yamaç, 08.08.2014, L8, O.Emin 22493\&Aksu, NE, Eux., Thp.

Lathraea squamaria L.; Yol kenarı, 17.05.2015, L6, O.Emin 22737\&Aksu, Euro-Sib., Hcrp.

Orobanche sp.

Yol kenarı, 17.05.2015, L4, O.Emin 22738\&Aksu, Hcrp.

Euphrasia pectinata Ten.

Açık alan, 08.08.2014, L7, O.Emin 22494\&Aksu, Euro-Sib.

Thp.

Bungea trifida (Vahl) C. A. Meyer
Yol kenarı, 02.04.2015, L2, O.Emin 22595\&Aksu, Hcrp. 63. VERBENACEAE

Verbena officinalis $\mathrm{L}$.

Dere yatağı, 08.08.2014, L4, O.Emin 22495\&Aksu, Cosm., Hcrp.

64. LAMIACEAE

Ajuga orientalis L.; Alpin vejetasyon, kayalık yamaç, 08.08.2014, L9, O.Emin 22496\&Aksu, Euro-Sib., Crp.

Teucrium orientale $\mathrm{L}$.

Yol kenarı, 28.07.2015, L8, O.Emin 22845\&Aksu, Ir-Tur. Hcrp.

T. chamaedrys $\mathrm{L}$.

Taşık yamaç, 28.07.2015, L11, O.Emin 22846\&Aksu, Hcrp.

Scutellaria orientalis L.

Taşlık alan, 17.05.2015, L6, O.Emin 22739\&Aksu, Ir-Tur., Hcrp.

Eremostachys moluccelloides Bunge

Taşlık alan, 17.05.2015, L1, O.Emin 22740\&Aksu, Hcrp.

Phlomis armeniaca Willd. ; Yol kenarı, taşlık kayalık alan, 17.05.2015, L1, O.Emin 22741\&Aksu, Endemik, LC, Ir-Tur. Hcrp.

Lamium galactophyllum Boiss. et Reuter; Çağıllık yamaç, 02.04.2015, L4, O.Emin 22596\&Aksu, Endemik, NE, Ir-Tur. Thp.

Ballota rotundifolia C. Koch; Kayalık yamaç, 28.07.2015, L5, O.Emin 22847\&Aksu, Endemik, NT, Ir-Tur. Hcrp.

Sideritis montana L.

Yol kenarı, 17.05.2015, L1, O.Emin 22742\&Aksu, Medit. Thp.

Stachys lavandulifolia Vahl.; Kayalık yamaç, 17.05.2015, L5, O.Emin 22743\&Aksu, Ir-Tur. Hcrp.

S. iberica M. Bieb. ; Orman içi açıklık, 08.08.2014, L12, O.Emin 22497\&Aksu, Ir-Tur., Hcrp.

S. annua (L.) L.

Yol kenarı, 08.08.2014, L5, O.Emin 22498\&Aksu, Cosm., Hcrp.

Nepeta supina Steven

Çağıllık yamaç, 28.07.2015, L3, O.Emin 22848\&Aksu, Horp.

Prunella vulgaris L.; Yol kenarı, 08.08.2014, L6, O.Emin 22499\&Aksu, Euro-Sib., Hcrp.

Origanum rotundifolium Boiss; Kayalık yamaç, 08.08.2014, L3, O.Emin 22500\&Aksu, NE, Eux., Chp.

Satureja hortensis L.; Kayalık yamaç, çağıllık alan, 08.08.2014, L1, O.Emin 22501\&Aksu, Chp.

Clinopodium vulgare L.; Taşlık yamaç, 08.08.2014, L10, O.Emin 22502\&Aksu, Euro-Sib., Hcrp.

Micromeria elliptica K. Koch; Kaya yarıkları, 17.05.2015, L12, O.Emin 22744\&Aksu, Endemik, LC, Ir-Tur. Hcrp. 
Thymus fallax Fisch. \& Mey.; Kayalık yamaç, 08.08.2014, L9, O.Emin 22503\&Aksu, Ir-Tur. Hcrp.

T. praecox Opiz subsp. grossheimii (Ronniger) Jalas var. grossheimii

Taşlık yamaç, 28.07.2015, L5, O.Emin 22849\&Aksu, NE, Chp.

Mentha longifolia (L.) L.; Yol kenarı, Islak yerler, 28.07.2015, L3, O.Emin 22850\&Aksu, LC, Eux., Crp.

M. spicata L.; Yol kenarı, Islak yerler, 28.07.2015, L1, O.Emin 22851\&Aksu, Crp.

Ziziphora tenuior L.;Kayalık yamaç, 17.05.2015, L1, O.Emin 22745\&Aksu, Ir-Tur. Thp.

Salvia rosifolia Sm.; Kayalık yamaç, 08.08.2014, L9, O.Emin 22506\&Aksu, Endemik, LC, Ir-Tur. Hcrp.

S. huberi Hedge; Taşlık yamaç, 17.05.2015, L1, O.Emin 22746\&Aksu, Endemik, LC, Ir-Tur. Hcrp.

S. microstegia Boiss. et Bal.; Kayalık yamaç, 17.05.2015, L4, O.Emin 22747\&Aksu, Ir-Tur. Hcrp.

S. limbata C. A. Mey.

Yol kenarı, 28.07.2015, L3, O.Emin 22852\&Aksu, Ir-Tur. Horp.

S. staminea Montbret \& Aucher ex Bentham; Kayalık yamaç, 08.08.2014, L9, O.Emin 22507\&Aksu, Ir-Tur. Hcrp. 65. PLUMBAGINACEAE

Acantholimon acerosum (Will.) Boiss. var. Acerosum; Kayalık yamaç, 06.08.2014, L1, O.Emin 22508\&Aksu, IrTur. Horp.

A. puberulum Boiss. \& Balansa; Çağıllık alan, 28.07.2015, L13, O.Emin 22853\&Aksu, Ir-Tur. Hcrp.

A. ulicinum (Willd. ex Schult.) Boiss

Taşlı yamaç, 28.07.2015, L8, O.Emin 22854\&Aksu, Hcrp.

66. PLANTAGINACEAE

Plantago major L. subsp. major; Yol kenarı, açık alan, 08.08.2014, L4, O.Emin 22509\&Aksu, Hcrp.

Veronica chamaedrys L.;Yol kenarı, 17.05.2015, L8, O.Emin 22748\&Aksu, Euro-Sib. Hcrp.

V. filiformis Sm.; Dere kenarı, 17.05.2015, L12, O.Emin 22749\&Aksu, Hyrc-Eux., Hcrp.

V. anagalis-aquatica L.; Açık ormanlık alan, 08.08.2014, L6, O.Emin 22510\&Aksu, LC, Hcrp.

V. liwanensis L.; Kayalık alan, 02.04.2015, L1, O.Emin 22597\&Aksu, VU, Euro-Sib. Hcrp.

V. multifida L.; Orman içi açık alan, 02.04.2015, L10, O.Emin 22598\&Aksu, Ir-Tur. Hcrp.

V. oltensis Woronow ex Elenevsky; Kayalık yamaç, 02.04.2015, L3, O.Emin 22599\&Aksu, Endemik, LC, Ir-Tur. Hcrp.

Globularia trichosantha Fisch. \& C. A. Mey Yol kenarı, 17.05.2015, L7, O.Emin 22750\&Aksu, Hcrp.

Linaria corifolia Desf.; Yol kenarı, 02.04.2015, L1, O.Emin 22600\&Aksu, Endemik, NE, Ir-Tur.
L. genistifolia (L.) Mill. subsp. confertiflora (Boiss.) Davis Kayalık yamaç, 28.07.2015, L5, O.Emin 22857\&Aksu, Endemik, LC, Ir-Tur. Hcrp.

\section{THYMELAEACEAE}

Daphne oleoides Screb.

Yol kenarı, 17.05.2015, L6, O.Emin 22751\&Aksu, Hcrp.

68.ELAEAGNACEAE

Elaeagnus angustifolia $\mathrm{L}$.

Yol kenarı, 17.05.2015, L2, O.Emin 22752\&Aksu, Php.

69. SANTALACEAE

Viscum album L. subsp. albüm; Pinus L. ormanı içinde, 28.07.2015, L5, O.Emin 22858\&Aksu, Vp.

Thesium arvense Horv.; Tarla alanı, 28.07.2015, L1, O.Emin 22859\&Aksu, Euro-Sib., Hcrp.

70. EUPHORBIACEAE

Chrozophora tinctoria (L.) A. Juss.

Taşlık, çakıllık yer, 28.07.2015, L1, O.Emin 22860\&Aksu, Thp.

Euphorbia peplis L.

Açık alan, 08.08.2014, L2, O.Emin 22511\&Aksu, Medit., Thp.

E. wittmannii Boiss.; Kaya çıkıntıları, 28.07.2015, L3, O.Emin 22861\&Aksu, Euro-Sib., Hcrp.

E. falcata $\mathrm{L}$.

Taşlık yamaç, 28.07.2015, L2, O.Emin 22862\&Aksu, Thp.

E. sp.

Çağıllık taşlık alan, 28.07.2015, L8, O.Emin 22863\&Aksu, Horp.

\section{PHYLLANTHACEAE}

Andrachne telephioides $\mathrm{L}$.

Yol kenarı, 17.05.2015, L1, O.Emin 22753\&Aksu, Hcrp.

72. URTICACEAE

Urtica dioica L.; Orman içi açıklıklarda, yol kenarı, 08.08.2014, L6, O.Emin 22512\&Aksu, Euro-Sib., Hcrp.

73. CANNABACEAE

Celtis iguanaea (Jacq.) Sarg.

Yol kenarı, 28.07.2015, L1, O.Emin 22855\&Aksu, Php.

74. ULMACEAE

Ulmus minör Mill. subsp. minör; Karışık yapraklı orman alanı, 08.08.2014, L6, O.Emin 22513\&Aksu, E. Medit., Php.

75. JUGLANDACEAE

Juglans regia $\mathrm{L}$.

Yol kenarı, 08.08.2014, L5, O.Emin 22514\&Aksu, Php.

\section{FAGACEAE}

\section{Fagus orientalis Lipsky}

Yaprak döken ve karışık ormanlık alan, nemli topraklarda, 08.08.2014, L10, O.Emin 22515\&Aksu, Euro-Sib., Php.

Quercus petraea (Mattuschka) Liebl. subsp. iberica (Steven ex Bieb.) Krassiln.

Yol kenarı, 08.08.2014, L5, O.Emin 22516\&Aksu, Php. 
Q. macranthera subsp. syspirensis (K. Koch) Menitsky; Kurak yamaçlar, 08.08.2014, L5, O.Emin 22517\&Aksu, Endemik, NE, Php.

\section{BETULACEAE}

\section{Carpinus betulus $\mathrm{L}$.}

Yol kenarı, 08.08.2014, L6, O.Emin 22518\&Aksu, Php.

LC, Euro-Sib., Php.

C. orientalis Mill.

Yol kenarı, 08.08.2014, L4, O.Emin 22519\&Aksu, LC, Php. Corylus avellana $\mathrm{L}$.

Yol kenarı, 17.05.2015, L3, O.Emin 22754\&Aksu, Eux., Chp.

Betula pendula Roth.

Yol kenarı, 08.08.2014, L10, O.Emin 22520\&Aksu, LC, Php. Alnus glutinosa (L.) Gaertn. subsp. barbata (C.A.Mey)

Yalt.

Dere kenarı, 17.05.2015, L5, O.Emin 22755\&Aksu.

NE, Eux., Php.

\section{SALICACEAE}

Salix triandra L.; Dere kenarı, 02.04.2015, L4, O.Emin 22601\&Aksu. Euro-Sib., Php.

S. alba L.; Yol kenarı, dere kenarı, 17.05.2015, L4,O.Emin 22756\&Aksu LC, Euro-Sib., Php

S. excelsa J. F. Gmelin; Dere kenarı, 08.08.2014, L2, O.Emin 22521\&Aksu, LC, Ir-Tur., Php.

S. caprea L. ; Dere kenarı, 17.05.2015, L3, O.Emin 22757\&Aksu,

Euro-Sib., Php.

S. wilhelmsiana M. Bieb.; Dere kenarı, 08.08.2014, L6,

O.Emin 22522\&Aksu, Ir-Tur., Php.

S. pedicellata Desf. subsp. pedicellata Desf.

Dere kenarı, 08.08.2014, L2, O.Emin 22523\&Aksu, Php.

Populus tremula L.; Karışık orman alanı, 08.08.2014, L12, O.Emin 22524\&Aksu, Euro-Sib.,Php.

79. RUBIACEAE

Asperula orientalis Boiss. \& Hohen. ; Yol kenarı, 08.08.2014, L12, O.Emin 22525\&Aksu, Ir-Tur., Thp.

Galium tricornutum $\mathrm{L}$.

Açık alan, 02.04.2015, L1, O.Emin 22602\&Aksu, Medit, Thp.

G. margaceum Ehrend. \& Schönb.-Tem; Kayalık yamaç, 17.05.2015, L4, O.Emin 22758\&Aksu, Endemik, LC, Thp.

Cruciata taurica (Pall. ex Willd.) Ehrend.; Yol kenarı, 17.05.2015, L8, O.Emin 22759\&Aksu, Ir-Tur., Chp.

Rubia peregrina $\mathrm{L}$.

Çalılık alan, 28.07.2015, L1, O.Emin 22856\&Aksu, Medit, Hcrp.

80. SMILACACEAE

Smilax excelsa L.; Karışık orman alanı, 17.05.2015, L3, O.Emin 22760\&Aksu, Eux., Chp.

\section{ASPARAGACEAE}

Scilla rosenii C. Koch.; Çayırlık alan, 02.04.2015, L7, O.Emin 22603\&Aksu, Eux., Crp.

S. monanthos C.Koch; Çayırlık alan, 02.04.2015, L6, O.Emin 22604\&Aksu, Eux., Crp.

S. siberica Haw. subsp. armena (Grossh.) Mordak; Çayırlık alan, 17.05.2015, L9, O.Emin 22761\&Aksu, Ir-Tur. Crp.

Ornithogalum narbonense $\mathrm{L}$.

Yol kenarı, 17.05.2015, L2, O.Emin 22762\&Aksu, Medit., Crp.

Muscari armeniacum Leichtlin ex Baker

Yol kenarı, 02.04.2015, L6, O.Emin 22605\&Aksu, Cosm. Crp.

Leopoldia tenuiflora (Tausch) Heldr.

Çayırlık alan, 17.05.2015, L4, O.Emin 22763\&Aksu, Crp.

82. LILIACEAE

Fritillaria pinardii Boiss.; Kayalık yamaç, 17.05.2015, L6, O.Emin 22764\&Aksu, Ir-Tur., Crp.

Gagea bohemica (Zauschn.) Schult. \& Schult. f.; Kayalık yamaç, 02.04.2015, L6, O.Emin 22606\&Aksu, Crp.

G. glacialis C. Koch; Çayırlık alan, 02.04.2015, L6, O.Emin 22607\&Aksu, Ir-Tur., Crp.

Tulipa armena Boiss.; Taşlık yamaç, 17.05.2015, L12, O.Emin 22765\&Aksu, Ir-Tur., Crp.

\section{XANTHORRHOEACEAE}

Asphodeline cena (Boiss.) Baker.; Dere kenarı, 17.05.2015, L1, O.Emin 22766\&Aksu, Ir-Tur., Crp.

\section{COLCHICACEAE}

Colchicum speciosum Steven.; Yol kenarı, 08.08.2014, L5, O.Emin 22526\&Aksu, Euro-Sib., Crp.

\section{AMARYLLIDACEAE}

Allium schoenoprasum L.; Kayalık-taşlık yamaç, 08.08.2014, L9

O.Emin 22527\&Aksu, Crp

A. zebdanense Boiss. \& Noe; Taşlı yamaç, 17.05.2015, L7, O.Emin 22767\&Aksu, Ir-Tur., Crp.

A. rotundum $\mathrm{L}$.

Yol kenarı, 08.08.2014, L7, O.Emin 22528\&Aksu, Eux., Crp.

A. sp.

Çağıllık alan, 28.07.2015, L8, O.Emin 22864\&Aksu, Crp.

86. IRIDACEAE

Crocus scharojanii Rupr.;Alpin vejeyasyon, 08.08.2014, L9, O.Emin 22529\&Aksu, VU, Eux., Hcrp.

C. vallicola Herbert; Alpin çayırlık, 08.08.2014, L7, O.Emin 22530\&Aksu, Eux., Crp.

Gladiolus atroviolaceus Boiss.; Picea orientalis orman alanı, 17.05.2015, L12, O.Emin 22768\&Aksu, Ir-Tur., Crp.

Irıs caucasica Hoffm.; Kayalık yamaç, 17.05.2015, L12, O.Emin 22769\&Aksu, Euro-Sib., Hcrp. 
I. nezahatiae Güner \& H. Duman; Kayalık yamaç, 17.05.2015, L4, O.Emin 22770\&Aksu, Endemik, CR, IrTur., Hcrp.

\section{ORCHIDACEAE}

Dactylorhiza romana (Seb.) Soo ; Çayırlık alan, 17.05.2015, L10, O.Emin 22771\&Aksu, Eux., Crp.

D. umbrosa (Kar. \& Kir.) Nevski; Çayırlık alan, 17.05.2015, L6, O.Emin 22772\&Aksu, Ir-Tur., Crp.

88. DIOSCOREACEAE

Dioscorea communis (L.) Caddick \& Wilkin; Karışık orman, 08.08.2014, L5, O.Emin 22531\&Aksu, Crp.

89. JUNCACEAE

Juncus inflexus $\mathrm{L}$.

Çayırlık alan, 08.08.2014, L7, O.Emin 22532\&Aksu, LC, Horp.

J.effusus L.; Dere kenarı, 17.05.2015, L2, O.Emin 22773\&Aksu,

LC, Cosm. Hcrp.

90. CYPERACEAE

Cyperus glaber $\mathrm{L}$.

Dere kenarı, 17.05.2015, L2, O.Emin 22774\&Aksu, LC, Crp.

Carex atrata L. ; Alpin çayırlık, 08.08.2014, L9, O.Emin 22533\&Aksu, Euro-Sib. (mt), Crp.

\section{POACEAE}

Brachypodium sylvaticum (Hudson) P. Beauv; Ormanlık yamaç, 08.08.2014, L12, O.Emin 22534\&Aksu, Euro-Sib., Hcrp.

B. pinnatum (L.) P. Beauv; Çayırlık yamaç, 08.08.2014, L11, O.Emin 22535\&Aksu, Euro-Sib., Crp.

Agropyron cristatum (L.) Gaertner

Çayırlık alan, 17.05.2015, L12, O.Emin 22775\&Aksu, Hcrp. Elymus repens (L.) Gould ; Kayalık-taşlık yamaç, 08.08.2014, L13, O.Emin 22536\&Aksu, Crp.

E. nodosus subsp. sinuatus (Nevski) Melderis; Kayalık yamaç, 17.05.2015, L6, O.Emin 22776\&Aksu, Ir-Tur., Hcrp.

Eremopyrum orientale (L.) Jaub. \& Spach; Dere kenarı, 02.04.2015, L4, O.Emin 22608\&Aksu, Ir-Tur, Thp.

Aegilops tauschii Cosson ; Açık alan, 08.08.2014, L2, O.Emin 22537\&Aksu, VU, Ir-Tur, Thp.

\section{A. triuncialis $\mathrm{L}$.}

Çayırlık alan, 08.08.2014, L7, O.Emin 22538\&Aksu, Thp.

Psathyrostachys fragilis (Boiss.) Nevski; Taşlık yamaç, 28.07.2015, L8, O.Emin 22865\&Aksu, Ir-Tur, Hcrp.

Bromus japonicus Thunb.

Yol kenarı, 08.08.2014, L2, O.Emin 22539\&Aksu, Thp.

B. armenus Boiss.; Alpin vejetasyon, 08.08.2014, L9, O.Emin 22540\&Aksu, Endemik, NT, Ir-Tur, Hcrp.
Avena fatua L. ; Çakıllı dere kenarı, 17.05.2015, L2, O.Emin 22777\&Aksu, Euro-Sib., Thp.

Helictotrichon argaeum (Boiss.) Parsa; Alpin vejetasyon, 08.08.2014, L9, O.Emin 22541\&Aksu, Endemik, LC, Ir-Tur, Horp.

Trisetum rigidum (M. Bieb.) Roemer \& Schultes; Dağ yamacı, 08.08.2014, L9, O.Emin 22542\&Aksu, Ir-Tur., Hcrp.

Agrostis canina L.

Dere kenarı, 08.08.2014, L3, O.Emin 22543\&Aksu, EuroSib.

A. gigantea Roth; Dere kenarı kumul alan, 08.08.2014, L2, O.Emin 22544\&Aksu, Euro-Sib., Crp.

A. balansae (Boiss.)Tzvelev; Çayırlık alan, 08.08.2014, L12, O.Emin 22545\&Aksu, NE, Euro-Sib (mt), Hcrp.

Phalaris arundinacea $\mathrm{L}$.

Dere kenarı, 17.05.2015, L9, O.Emin 22867\&Aksu, LC Crp.

Phleum alpinum L.; Alpin vejetasyon, 08.08.2014, L9, O.Emin 22546\&Aksu, LC, Euro-Sib., Crp.

P. paniculatum Hudson; Kayalık yamaç, 08.08.2014, L8, O.Emin 22547\&Aksu, Ir-Tur., Thp.

P. exaratum Griseb ; Açık alan, 17.05.2015, L8, O.Emin 22778\&Aksu, Cosm., Thp.

Festuca gigantea (L.) Vill.; Kayalık alan, 08.08.2014, L8, O.Emin 22548\&Aksu, Euro-Sib., Chp.

F. drymeja Mert. \& Koch; Orman alanı, 08.08.2014, L3, O.Emin 22549\&Aksu, Euro-Sib., Hcrp.

\section{F. arundinaceae Schreber}

Çayırlık alan, 08.08.2014, L7, O.Emin 22550\&Aksu, Chp. F. valesiaca Schleicher ex Gaudin

Alpin vejetasyon, 08.08.2014, L9, O.Emin 22551\&Aksu, Chp.

Lolium persicum Boiss. et Hohen ex Boiss.; Açık çayırlık alan, 08.08.2014, L7, O.Emin 22552\&Aksu, Iran-Tur, Thp.

L. rigidum Gaudin

Yol kenarı, 08.08.2014, L3, O.Emin 22553\&Aksu, Thp.

Poa supina Schrader; Alpin vejetasyon, 08.08.2014, L9, O.Emin 22554\&Aksu, Euro-Sib, Hcrp.

P. trivialis L.; Dere kenarı çayırlık alan, 08.08.2014, L6, O.Emin 22555\&Aksu, Chp.

P. pratensis L. ; Alpin vejetasyon, 08.08.2014, L9, O.Emin 22556\&Aksu, Cosm., Crp.

P. angustifolia L.; Pinus L. ormanı, 17.05.2015, L3, O.Emin 22779\&Aksu, LC, Cosm., Crp.

P. caucasica Trin; Dere kenarı, 08.08.2014, L8, O.Emin 22556\&Aksu, VU, Eux, Hcrp.

P. alpina $\mathrm{L}$.

Kayalık alan, 08.08.2014, L8, O.Emin 22557\&Aksu, Chp. P. bulbosa L. 
Alpin vejetasyon, 08.08.2014, L9, O.Emin 22558\&Aksu, Chp.

P. nemoralis L.

Dere kenarı, 08.08.2014, L10, O.Emin 22559\&Aksu, Chp. Sclerochloa dura (L.) P. Beauv.; Dere kenarl, 17.05.2015, L3, O.Emin 22780\&Aksu, Euro-Sib., Thp.

Dactylis glomerata L.subsp hispanica (Roth.) Nyman Ormanlık alan, 08.08.2014, L7, O.Emin 22560\&Aksu, Crp.

Cynosurus cristatus L. ; Picea orientalis orman alanı, 08.08.2014, L6, O.Emin 22561\&Aksu, Euro-Sib., Hcrp.

C. echinatus $L$.

Yol kenarı, 17.05.2015, L3, O.Emin 22781\&Aksu, Medit., Thp.

Briza media L.

Nemli yamaç, 08.08.2014, L12, O.Emin 22562\&Aksu, Crp.

\section{B. minor $\mathrm{L}$.}

Yol kenarı, 17.05.2015, L2, O.Emin 22782\&Aksu, Thp.

Echinaria capitata (L.) Desf.

Taşlı yamaç, 02.04.2015, L2, O.Emin 22609\&Aksu, Thp.

Melica uniflora Retz.; Ormanlık alan, 08.08.2014, L5, O.Emin 22563\&Aksu, Euro-Sib., Crp.

M. ciliata L. ; Alpin vejetasyon, kayalık alan, 08.08.2014, L9, O.Emin 22564\&Aksu, Hcrp.

M. persica Kunth

Taşlık yamaç, 08.08.2014, L9, O.Emin 22565\&Aksu, Hcrp.

Molina caerulea (L.) Moench

Ormanlık alan, 08.08.2014, L5, O.Emin 22566\&Aksu, Crp.

Stipa bromoides (L.) Dörfler

Açık alan, 17.05.2015, L2, O.Emin 22783\&Aksu, Medit., Crp.
Phragmites australis (Cav.) Trin. ex Steudel; Dere kenarı, 08.08.2014, L6, O.Emin 22567\&Aksu, LC, Euro-Sib, Hcrp. Danthonia decumbens (L.) DC.

Ormanlık alan, 08.08.2014, L7, O.Emin 22568\&Aksu, Hcrp.

Eragrostis minor Host; Kayalık-taşlık yamaç, , 08.08.2014, L8, O.Emin 22569\&Aksu, Thp.

Crypsis schoenoides (L.) Lam.; Çakıl taşlı dere kenarı, 08.08.2014, L2, O.Emin 22570\&Aksu, LC, Thp.

Tragus racemosus (L.) All.

Çakıllı dere kenarı, 08.08.2014, L6. O.Emin 22571\&Aksu, Thp.

Pennisetum orientale L. C. M. Richard; Kayalık alan, 08.08.2014, L7, O.Emin 22572\&Aksu, Ir-Tur, Hcrp.

Sorghum halepense (L.) Pers.

Dere kenarı, 08.08.2014, L4, O.Emin 22573\&Aksu, Hcrp.

Bothriochloa ischaemum (L.) Keng

Dere kenarı, 08.08.2014, L2, O.Emin 22574\&Aksu, Hcrp.

\section{TARTISSMA ve SONUÇ}

Araştırma alanında teşhis edilen 493 taksonun, taksonomik birimlere dağılımı ve endemizm durumu Çizelge 2'de verilmiştir. Türkiye florası, bu alanda $\% 4.2$ oranında temsil edilmektedir. Ülkemizde 3649 takson endemik olup endemizm oranı \%31.82'dir (Güner vd 2012). Avrupa-Sibirya fitocoğrafik bölgesinde yaklaşık 300 takson (Ekim vd 2000), Doğu Karadeniz Bölgesi için 220 takson endemik olup endemizm oranı \%9.8'dir (Anşin, 1982).

Çizelge 2. Saptanan taksonların taksonomik birimlere dağılımı.

\begin{tabular}{llllllll}
\hline & Familya & Cins & Tür & subsp. & var. & Takson & Endemik \\
\hline Lycopodiophyta & 1 & 1 & 1 & - & - & 1 & - \\
Pteridophyta & 9 & 10 & 17 & 1 & - & 18 & - \\
Magnoliophyta & 81 & 320 & 420 & 41 & 13 & 474 & 40 \\
Pinophytina & 2 & 4 & 3 & 2 & 2 & 7 & - \\
Magnoliophytina & 79 & 316 & 417 & 39 & 11 & 467 & 40 \\
Toplam & $\mathbf{9 1}$ & $\mathbf{3 3 1}$ & $\mathbf{4 3 8}$ & $\mathbf{4 2}$ & $\mathbf{1 3}$ & $\mathbf{4 9 3}$ & $\mathbf{4 0}$ \\
\hline
\end{tabular}

Araştırma alanında saptanan 40 takson endemik olup endemizm oranı \%8.1'dir. Bitkilerin endemizm durumu, fitocoğrafik bölgelere göre dağılımları ve IUCN kategorileri Çizelge 3'te verilmiştir. Araştırma alanı, bitki coğrafyası yönünden Holarktik bölgenin, Avrupa-Sibirya fitocoğrafik alanının Öksin kesiminin Kolşik altkesiminde kalmaktadır.
Araştırma alanında saptanan 91 familya içerisinde en fazla takson içeren familyalara ilişkin taksonların ve cinslerin sayısal ve oransal dağılımları Çizelge 4'de, taksonların cinslere göre sayısal dağılımı ise Çizelge 5'de verilmiştir verilmiştir. 
Çizelge 3. Saptanan endemik ve endemik olmayan taksonların fitocoğrafik bölgelere ve tehlike kategorilerine dağılımı

\begin{tabular}{lllllll}
\hline $\begin{array}{l}\text { Fitocoğrafik } \\
\text { bölge }\end{array}$ & \multicolumn{2}{l}{ Endemik } & \multicolumn{3}{l}{$\begin{array}{l}\text { Endemik } \\
\text { olmayan }\end{array}$} & \multicolumn{2}{l}{ Toplam } \\
\hline & Sayı & $\%$ & Sayı & $\%$ & Sayı & $\%$ \\
Euro Sib. & 3 & 0.6 & 127 & 25.8 & 130 & 26.4 \\
Ir-Tur. & 32 & 6.5 & 61 & 12.4 & 93 & 18.9 \\
Medit. & 2 & 0.4 & 22 & 4.5 & 24 & 4.9 \\
Kosm. ve diğer & 3 & 0.6 & 244 & 49.2 & 247 & 49.8 \\
Toplam & $\mathbf{4 0}$ & $\mathbf{8 . 1}$ & $\mathbf{4 5 3}$ & $\mathbf{9 1 . 9}$ & $\mathbf{4 9 3}$ & $\mathbf{1 0 0}$ \\
CR & 2 & 0.4 & - & - & 2 & 0.4 \\
EN & 5 & 1 & 1 & 0.2 & 6 & 1.2 \\
VU & 3 & 0.6 & 7 & 1.4 & 10 & 2 \\
NT & 3 & 0.6 & 1 & 0.2 & 4 & 0.8 \\
LC & 18 & 3.7 & 31 & 6.3 & 49 & 10 \\
NE & 9 & 1.8 & 10 & 2 & 19 & 3.8 \\
\hline Toplam & $\mathbf{4 0}$ & $\mathbf{8 . 1}$ & $\mathbf{5 0}$ & $\mathbf{1 0 . 1}$ & $\mathbf{9 0}$ & $\mathbf{1 8 . 2}$ \\
\hline
\end{tabular}

Çizelge 4. Araştırma alanında saptanan cins ve taksonların familyalara göre sayısal dağılımı

\begin{tabular}{lllll}
\hline Familya & $\begin{array}{l}\text { Cins } \\
\text { sayısı }\end{array}$ & $\mathbf{( \% )}$ & $\begin{array}{l}\text { Takson } \\
\text { sayısı }\end{array}$ & $\mathbf{( \% )}$ \\
\hline Asteraceae & 42 & 12.68 & 60 & 12,2 \\
Poaceae & 32 & 9.66 & 55 & 11,2 \\
Fabaceae & 18 & 5.43 & 32 & 6,5 \\
Lamiaceae & 19 & 5.74 & 28 & 5,7 \\
Rosaceae & 12 & 3.62 & 21 & 4,3 \\
Boraginaceae & 14 & 4.22 & 19 & 3,9 \\
Apiaceae & 16 & 4.83 & 18 & 3,7 \\
Brassicaceae & 15 & 4.53 & 18 & 3,7 \\
Caryophllaceae & 7 & 2.11 & 11 & 2,2 \\
Plantaginaceae & 4 & 1.20 & 10 & 2,4 \\
Caprifoliaceae & 9 & 2.71 & 9 & 1,8 \\
Orobanchaceae & 7 & & 9 & 1,8 \\
Diğer & 141 & & 201 & \\
\hline
\end{tabular}

Çizelge 5. Araştırma alanında en fazla tür ve türaltı taksona sahip cinsler

\begin{tabular}{lll}
\hline Cins & Takson Sayısı & $\%$ \\
\hline Poa & 8 & 1,6 \\
Trifolium & 7 & 1,4 \\
Campanula & 6 & 1,2 \\
Veronica & 6 & 1,2 \\
Salix & 6 & 1,2 \\
Salvia & 5 & 1 \\
Psephellus & 5 & 1 \\
Geranium & 5 & 1 \\
Diğer & 445 & 90,3 \\
\hline
\end{tabular}

Çalışma kapsamında alanda tespit edilen cins sayısına göre en zengin ilk beş familya: Asteraceae (42), Poaceae (32), Lamiaceae (19), Fabaceae (18) ve Apiaceae (16). Takson sayısına göre en zengin ilk beş familya ise Asteraceae (60), Poaceae (55), Fabaceae (32), Lamiaceae (28), ve Rosaceae (21). Takson sayısına göre alanda tespit edilen en zengin cinsler: Poa (8), Trifolium (7), Campanula
(6), Veronica (6), Salix (6), Salvia (5), Psephellus (5) ve Geranium (5).

Araştırma alanında yer alan en fazla türe sahip cinsin Poa olması, Karadağ bölgesinde ormanlık alanın azlığı, açık mera ve alpin alanlarının bulunduğunun göstergesidir. Artvin ilinde gözlenen üç iklim tipinden biri olan oseionik iklimin gösterge cinsi olan Trifolium'da alanda en fazla tür içeren ikinci tür olarak gözlenmiştir. Bu cins nemli serin yerlerde yetişmektedir. Campanula ise ılıman iklim ve Akdeniz ikliminin hüküm sürdüğü alanlarda gözlenen bir bitki cinsidir. Alanda görülen iklim tipleri vejetasyon üzerine etkili olmuştur.

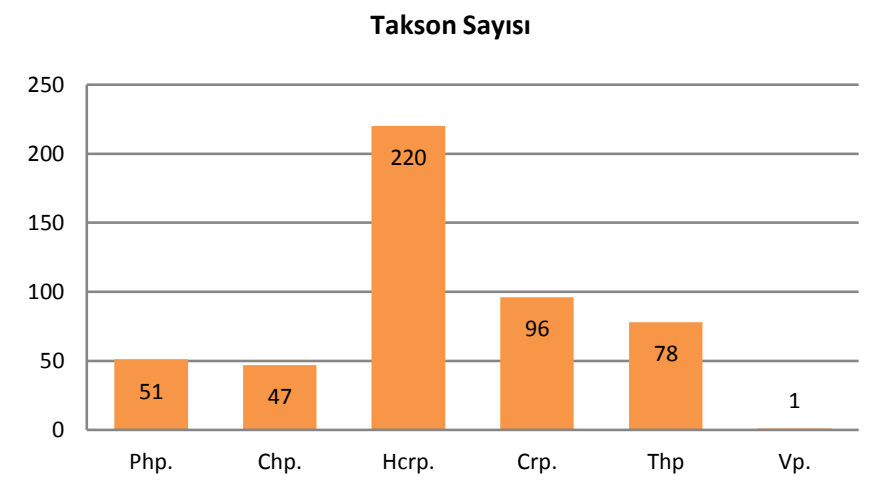

Şekil 3. Tespit edilen taksonların Raunkier (1934)'e göre yaşam formlarının dağılımı

Çalışma alanından tespit edilen taksonların Raunkiær (1934)'e göre düzenlenen yaşam formları Şekil 3'de verilmiştir. Elde edilen verilere göre tespit edilen bitki taksonlarının 220 (\%44) hemikriptofit, 96 (\%19) kriptofit, 78 (\%16) terofit, 51 (\%10) fanerofit, 47 (\%10) kamefit ve $1(\% 0.4)$ vasküler parazit takson tespit edilmiştir. En fazla yayılış gösteren bitki formları kriptofit olduğu tespit edilmiştir. Yakın alanlarda yapılan diğer çalışmalarla fitocoğrafik bölge elementlerinin oransal dağılımının karşılaştırılması Çizelge 6 'da verilmiştir. Araştırma alanı Avrupa-Sibirya fitocoğrafik bölgesi içerisinde kaldığından dolayı Avrupa-Sibirya kökenli taksonların oranı daha yüksektir. Ancak Ocakverdi (2001)'in A9 karesinin İranTuran fitocoğrafik bölgesinde kalan çalışmasında İranTurhan kökenli bitkilerin oranı daha yüksektir. Araştırma alanı A9 karesi içerisinde yer almakta olup Avrupa-Sibirya fitocoğrafik bölgesi ile Iran-Turan fitocoğrafik bölgesi arasında geçiş bölgesinde bulunduğundan Iran-Turan kökenli bitkilerin oranı diğer çalışmalara göre daha yüksektir. 
Çizelge 6. Fitocoğrafik bölge elementlerinin ve endemizm oranının yakın bölgelerde yapılan çalışmalarla karşılaştırılması (\%).

\begin{tabular}{lllllll}
\hline Çalışmalar & Takson Sayısı & Euro-Sib. & Ir-Tur. & Medit. & Cosm. ve diğer & Endemizm \\
\hline Karadağ & 493 & 26,4 & 18,9 & 4,9 & 49,8 & 8,1 \\
Eminağaoğlu ve ark. 2015a & 2727 & 22,3 & 13,3 & 1,7 & 62,5 & 7,2 \\
Eminağaoğlu ve Aksu, 2015 & 593 & 39,6 & 7,6 & 1,9 & 50,9 & 7,1 \\
Eminağaoğlu ve ark. 2008 & 990 & 48,2 & 3,5 & 1,9 & 46,4 & 2,3 \\
Eminağaoğlu ve ark. 2007 & 963 & 48,9 & 2 & 2 & 47,1 & 1,1 \\
Eminağaoğlu ve ark. 2004 & 872 & 39,4 & 10,3 & 1,2 & 49,1 & 7,3 \\
Eminağaoğlu ve ark. 2003 & 769 & 35,6 & 6,9 & 2,2 & 55,3 & 7,4 \\
Ocakverdi 2001 & 577 & 22.3 & 22.5 & - & 14,4 & 5,5 \\
Anşin 1979 & 578 & 22,3 & 22,5 & - & 55,2 & \\
\hline
\end{tabular}

Tespit edilen taksonlardan 247 tanesinin fitocoğrafik bölgelere göre dağılımları ise şöyledir: 130 adedi AvrupaSibirya, 93 adedi İran-Turan, 24 adedi Akdeniz kökenlidir. Endemik ve endemik olmayan nadir bitkiler uluslararası IUCN tehlike kategorilerine göre sınıflandırılmıştır. Araştırma alanında bulunan nadir bitkilerin IUCN kategorilerine göre dağııımı; 2 endemik takson CR, 5 endemik ve 1 endemik olmayan takson EN, 3 endemik ve 7 endemik olmayan takson $\mathrm{VU}, 3$ endemik ve 1 endemik olmayan takson NT, 18 endemik ve 31 endemik olmayan takson LC ve 9 endemik ve 10 endemik olmayan takson NE.

Araştırma alanında tespit edilen 91 familya içerisinden en fazla takson içeren 5 familyanın yakın bölgelerde yapılan çalışmalarla oransal karşılaştırmaları Çizelge 7'de verilmiştir. Türkiye Florası'nda en fazla takson içeren familyalar; Asteraceae, Fabaceae, Lamiaceae, Poaceae,
Brassicaceae, Caryophyllaceae, Scrophulariaceae, Apiaceae, Liliaceae, Boraginaceae, Rosaceae, Campanulaceae, Rubiaceae'dir (Davis 1988). Yakın bölgelerde yapılan diğer çalışmalarda da; en fazla tür içeren familyaların Asteraceae, Fabaceae, Lamiaceae, Poaceae ve Rosaceae olduğu belirlenmiştir (Anşin, 1999; Ocakveri, 2001; Eminağaoğlu vd., 2018). Bu çalışmalar, araştırma alanından elde edilen sonuçlarla benzerlik göstermektedir. Araştıma alanının büyük bir bölümünün step ve yüksek dağ çayırlarından oluşması, karasal iklim etkisi altında olması ve ayrıca yüksek eğim ve sıcaklığa bağlı verimsiz ve sığ topraklar Asteraceae, Poaceae ve Fabaceae familyasına ait bitki türlerinin bolluğuna sebep olmuştur. Çalışma alanları benzer coğrafik ve ekolojik özellikler gösterdiğinden bu çalışma sonucu elde edilen bitkilerin dağılım oranları diğer bölgelerle benzerlik göstermiştir.

Çizelge 7. En çok takson içeren 8 familyanın yakın bölgelerde yapılan çalışmalar ile oransal karşılaştırılması (\%).

\begin{tabular}{|c|c|c|c|c|c|c|}
\hline Familyalar & Taxon & Asteraceae & Poaceae & Fabaceae & Lamiaceae & Rosaceae \\
\hline Karadağ & 493 & 12,2 & 11,2 & 6,5 & 5,7 & 4,3 \\
\hline Eminağaoğlu ve ark. 2015a & 2727 & 13,6 & 7,15 & 7,44 & 5,9 & 5,2 \\
\hline Eminağaoğlu ve Aksu 2015 & 593 & 11 & 10,1 & 4,7 & 5,2 & 5,7 \\
\hline Eminağaoğlu ve ark. 2008 & 990 & 11,5 & 7 & 6 & 4,3 & 6 \\
\hline Eminağaoğlu ve ark. 2007 & 963 & 11,5 & 7 & 6 & 4,5 & 6,1 \\
\hline Eminağaoğlu ve ark. 2004 & 872 & 10,2 & 7 & 8,9 & 5,9 & 6,5 \\
\hline Eminağaoğlu ve ark. 2003 & 769 & 9,5 & 4,9 & 8,7 & 5,5 & 6,5 \\
\hline Ocakverdi 2001 & 577 & 16,8 & 7,3 & 7,3 & 5,7 & 5,7 \\
\hline Anşin 1979 & 578 & 8,9 & 4,0 & 8,1 & 4,7 & 5,6 \\
\hline
\end{tabular}

Araştırma alanında, uluslararası IUCN tehlike kategorilerinden özellikle EN, VU, CR kategorilerine dahil çok sayıda endemik ve endemik olmayan nadir bitkilerin bulunduğu görülmüştür. Bu biyolojik zenginlik dikkate alındığında araştırma alanının korunması gerekliliği ortaya çıkmaktadır.
Artvin ilindeki biyolojik çeşitlilik ve ekolojik bütünlük çeşitli etkenlerin yoğun tehdidi altındadır: Yatırım projeleri, baraj inşaatları, madencilik, kaçak kesim, yol yapımı, tarım alanlarının genişletilmesi, turizm ve yurt dışına ihraç ve yurt içi kullanım amacı ile doğadan bitki toplamaları sayılabilir. Araştırma alanı da bu etkenlerden birkaçına (kömür madenciliği, baraj inşaatları vb.) maruz kalmış durumdadır. Bu nedenle Karadağ ve çevresinde 
yapılacak benzeri faaliyetler alanı ekolojik açıdan etkileyip habitat değişimlerine yol açarak alandaki nadir ve endemik özellikli türlerin yok olmasına neden olabilecektir. $\mathrm{Bu}$ alanların korunup, izlenmesi gerekmektedir.

Bozulmamış doğal alanlarda en fazla zarar veren insan faaliyetleri yol açma çalışmalarıdır. Özellikle ekosistemleri küçük parçalara bölmesi ve doğrudan habitat kaybına yol açması, yol çalışmalarının çok dikkatle değerlendirilmesini gerekli kılmaktadır.

Alanda gerçekleştirilen kömür madenciliği bu bölgenin biyoçeşitliliğini tehlike altına sokmaktadır. Kömür işletmelerinin tatlı su kaynaklarını azaltması ve erozyona neden olması gibi nedenler ile bölgede yetişen endemik ve endemik olmayan birçok bitkinin gelişimini engelleyebilir, gelişim bozukluklarına yol açabilir ve bu bitkilerin bölgedeki yayılışını sonlandırabilir. Alanın çevresinde yapımı devam eden baraj inşaatı varolan bitkisel çeşitliliği doğrudan ve dolaylı olarak tehdit etmektedir. Baraj suları altında kalcak veya baraj yapımı nedeniyle oluşacak iklimsel değişimler nedeniyle zarar görecek popülasyonların ex-situ koruma ile belirlenen alanlara taşınımı ve yetiştirilmesi gerekmektedir. Gerçekleştirilen madencilik çalışmalarının denetimlerinin düzenli olarak yapılması, gereğinden fazla alanda madencilik faaliyetlerine başlanmaması gerekmektedir.

Çalışma alanında saptanan endemik ve endemik olmayan nadir türlerden yüksek IUCN risk kategorisine sahip olan özellikle Phlomis armeniaca, Iris caucasica, I. nezahatiae, Lathyrus woronowii, Genista aucheri, Uechtritzia armena, Micromeria elliptica, Lamium galactophyllum ve Tchihatchewia isatidea türleri alanda gerçekleştirilebilecek madencilik faaliyetlerinden etkilenebilecek ve nesilleri tehlike altına girebilecek türlerdir (Şekil 4). Daha düşük rakımlarda yayılış gösteren, popülasyonlarının büyük çoğunluğu baraj su aynası altında kalacak olan endemik bitki türlerinden Chesneya elegans, Convolvulus pseudoscammonia ve Bupleurum schistosum yol ve baraj yapımı faaliyetlerinden zarar görebilirler. Bu nedenle özellikle nadir ve endemik bu bitki türlerinin yayılış gösterdiği alanlar koruma altına alınarak nesillerinin devamı güvence altına alınmalıdır.

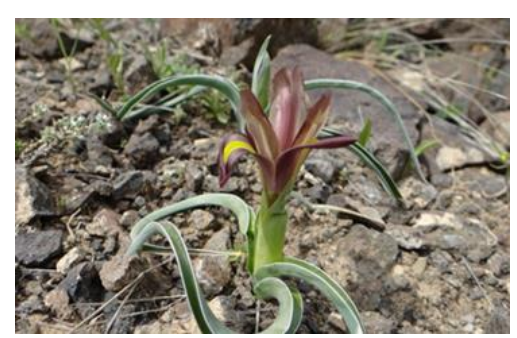

Iris nezahatiae

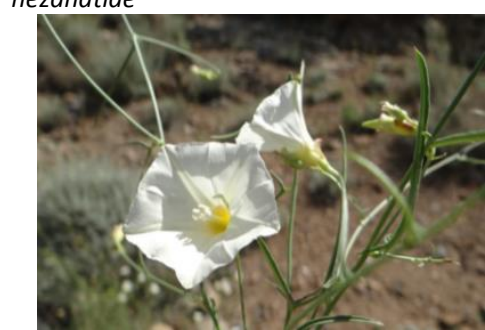

Convolvulus pseudoscammonia

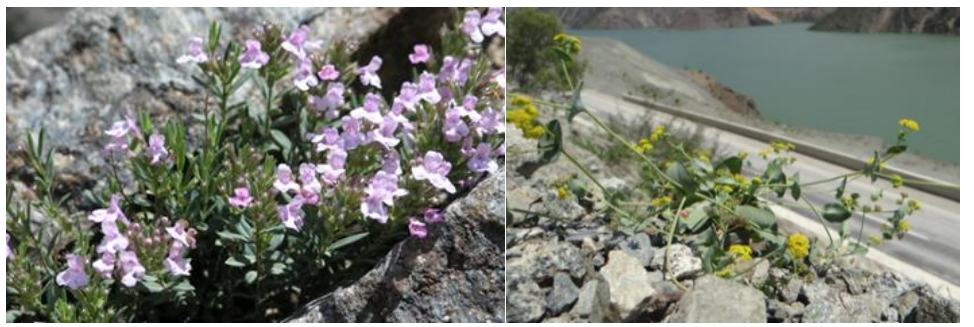

Bupleurum schistosum

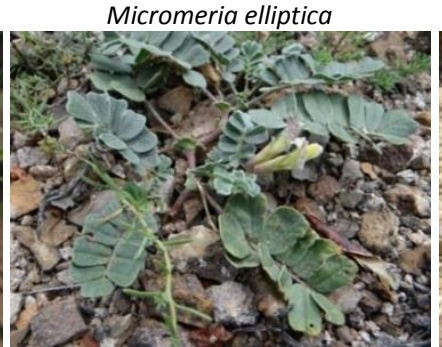

Chesneya elegans

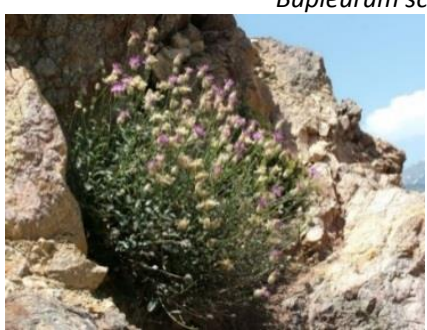

Psephellus pecho

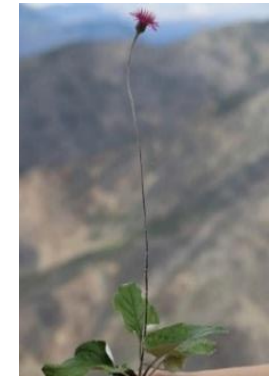

Uechtritzia armena

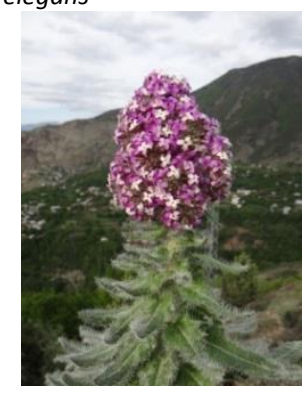

Tchihatchewia isatidea

Șekil 4. Bazı önemli endemik türler 


\section{KAYNAKLAR}

Akkemik Ü (ed) (2014) Türkiye'nin Doğal-Egzotik Ağaç ve Çalıları, Vol.12. Orman Genel Müdürlüğü Yayınları, Ankara.

Akman Y (1990) Iiklim ve Biyoiklim, Palme Yayınları, Ankara.

Anşin R (1979) Trabzon-Meryemana Araştırma Ormanı Florası ve Saf Ladin Mefşcerelerinde Floristik Arafştırmalar (Flore de la foret de Recherches de Meryemana-Trabzon et Les Recherches Floristiques Dans les Peuplements Purs D'Epicea). Trabzon: Karadeniz Gazetecilik ve Matbaacılık.

Anşin R (1982) Endemizm ve Doğu Karadeniz Bölgesinde Yetişen Endemik Bitki Taksonları, (Endemism And Endemic Plants Of The East Black Sea Region). KTÜ Orman Fakültesi Dergisi 5(2): 311-326

Davis PH (ed) (1965-1985) Flora of Turkey and the East Aegean Islands, Vols. 1-9. Edinburgh University Press, Edinburgh.

Davis PH, Mill RR, Tan K (1988) Flora of Turkey and the East Aegean Islands, Vol. 10. Edinburgh University Press, Edinburgh.

DMi (2015) T.C. Çevre ve Orman Bakanlığı Devlet Meteoroloji İşleri Genel Müdürlügü, Artvin 2003-2013 İklim Verileri, Ankara.

Düzenli A (1988) Neuve aux Sintaxons Phytosociologies pour la Végétation de Turquie. Ecol Med 14: 143-148.

Ekim T, Koyuncu M, Vural M, Duman H, Aytaç Z, Adıgüzel N (2000) Türkiye Bitkileri Kırmızı Kitabı, Eğrelti ve Tohumlu Bitkiler (Red Data Book of Turkish Plants, Pteridophyta and Spermatophyta). Barışcan Ofset, Ankara, pp 246

Ekim T, Terzioğlu S, Eminağaoğlu Ö, Coşkunçelebi K (2014) Turkey. In: J. Solomon, T. Schulkina \& G.E. Schatz (editors), Red List of the Endemic Plants of Caucasus: Armenia, Azerbaijan, Georgia, Iran, Russia, and Turkey. Monographs in Systematic Botany from the Missouri Botanical Garden (MSB) 125. Missouri Botanical Garden Press, Saint Louis, s:209-242.

Eminağaoğlu Ö (1996) Artvin-Atila (Hatilla) Vadisi Florası, Karadeniz Teknik Üniversitesi Fen Bilimleri Enstitüsü, Yükseklisans Tezi, Trabzon.

Eminağaoğlu Ö (2002) Şavşat ilıçesi Karagöl-Sahara Milli Parkı ve Çevresinin Flora ve Vejetasyonu, Karadeniz Teknik Üniversitesi Fen Bilimleri Enstitüsü, Doktora Tezi, Trabzon.

Eminağaoğlu Ö (2009) The Plant Diversity of Tekkale-Çevreli and Cemketen Villages (Yusufeli, Artvin). Batumi Botanical Garden Bulletin 33: 152-159.

Eminağaoğlu Ö (2012) Artvin'de Doğa Mirası-Camili'nin Doğal Bitkileri. Promat, ìstanbul.

Eminağaoğlu Ö (Ed) (2015) Artvin' in Doğal Bitkileri. Promat, İstanbul.

Eminağaoğlu Ö, Akpulat H.A (2010) Vaccinium myrtillus var. artvinense-A new taxon for the flora of Turkey. Annals of Agrarian Science 8 (1): 142-144.

Eminağaoğlu Ö, Aksu G (2015) Barhal Vadisi (Yusufeli, Artvin-Turkiye) Florası. Artvin Çoruh Üniversitesi Bilimsel Araştırma Projesi, AÇÜBAP 2013.F10.01.04

Eminağaoğlu Ö, Akyıldırım Beğen H, Aksu G (2015a) Artvin'in Flora ve Vejetasyon Yapısı, s 27-51. Şu eserde: Eminağaoğlu, Ö. (Ed.), Artvin' in Doğal Bitkileri. İstanbul: Promat
Eminağaoğlu Ö, Akyıldırım Beğen H, Aksu G (2015b) Artvin'in Genel Tanıtımı, s 1-25. Şu eserde: Eminağaoğlu, Ö. (Ed.), Artvin' in Doğal Bitkileri. İstanbul: Promat.

Eminağaoğlu Ö, Anşin R (2003) The Flora of Hatila Valley National Park and its Close Environs (Artvin). Turkish Journal of Botany 27 (1):127.

Eminağaoğlu Ö, Anşin R (2004) Flora of the Karagöl-Sahara National Park (Artvin) and Its Environs. Turkish Journal of Botany 28 (6): 557-590.

Eminağaoğlu Ö, Kutbay HG, Bilgin A, Yalçın E (2006) Contribution to the Phytosociology and Conservation of Tertiary Relict Species in the Northeastern Anatolia Region (Turkey). Belgian Journal of Botany 139 (1): 124-130.

Eminağaoğlu Ö, Kutbay HG, Özkan ZC, Ergül A (2008) Flora of the Camili Biosphere Reserve Area (Borçka, Artvin, Turkey). Turkish Journal of Botany 32 (1): 43-90.

Eminağaoğlu Ö, Özkaya MS, Akpulat HA (2012) A new record fort the flora of Turkey: Sorbus caucasica var. caucasica (Rosaceae). Turkish Journal of Botany 36: 426.

Eminağaoğlu Ö, Yüksek T, Gümüş S, Kurdoğlu O, Eraydın S (2007) Borçka-Karagöl Tabiat Parkı ve Çevresinin Flora ve Vejetasyonu. TÜBiTAK (Proje No:1030079), Ankara.

Eminağaoğlu Ö. Yüksel E, Akyıldırım Beğen H (2018) Flora of the Hod Valley (Artvin, Turkey). International Journal of Ecosystems and Ecology Science 8 (2): 273-282.

Eminağaoğlu Ö. Anşin R (2002) A9 (Artvin) Karesi İçin Yeni Floristik Kayıtlar. Kafkas Üniversitesi Artvin Orman Fakültesi Dergisi 3: 96108

Grossheim A A (1939-1967) Flora Kavkaza. Ciltler 1-7, Bakü ve Leningrad.

Güner A, Aslan S, Ekim T, Vural M, Babaç MT (2012) Türkiye Bitkileri Listesi (Damarlı Bitkiler). Nezahat Gökyiğit Botanik Bahçesi ve Flora Araştırma Derneği Yayını, İstanbul.

Güner A, Özhatay N, Ekim T, Başer KHC (2000) Flora of Turkey and the East Aegaen Islands, Vol. XI. Supplement - II, Edinburgh University Press, Edinburgh.

IUCN (2017) The IUCN Red List of Threatened Species. Version 2017-2. <http://www.iucnredlist.org>. Downloaded on 14 September 2017.

Komarov VL (1934-78) Flora of the U.S.S.R., Vol. 1-30. Israel Program for Scientific Translations, Jerusalem.

Ocakverdi H (2001) The Flora of the Mount Kısır (Kars and Ardahan) and Nearest Environs, Turkish Journal of Botany 25: 311-334.

Raunkiær C (1934) The Life Forms of Plants and Statistical Plant Geography, being the collected papers of C. Raunkiær. Translated by H. Gilbert-Carter, A. Fausbøll, and A. G. Tansley. Oxford University Press, Oxford.

The Plant List (2017) Version1. Published on the internet, http:// www. theplantlist.org/24.09.2015.

Vural M (1996) Rize'nin Yüksek Dağ Vejetasyonu (High mountain vegetation of Rize). Turkish Journal of Botany 20: 83-102. 